\title{
THE CONNECTION BETWEEN REDDENING, GAS COVERING FRACTION, AND THE ESCAPE OF IONIZING RADIATION AT HIGH REDSHIFT*
}

\author{
Naveen A. Reddy ${ }^{1,7}$, Charles C. Steidel ${ }^{2}$, Max Pettini ${ }^{3}$, Milan Bogosavljević ${ }^{4,5}$, and Alice E. Shapley ${ }^{6}$ \\ ${ }^{1}$ Department of Physics and Astronomy, University of California, Riverside, 900 University Avenue, Riverside, CA 92521, USA \\ ${ }^{2}$ Department of Astronomy, California Institute of Technology, MS 105-24, Pasadena, CA 91125, USA \\ ${ }^{3}$ Institute of Astronomy, Madingley Road, Cambridge CB3 OHA, UK \\ ${ }_{5}^{4}$ Astronomical Observatory, Belgrade, Volgina 7, 11060 Belgrade, Serbia \\ ${ }^{5}$ New York University Abu Dhabi, P.O. Box 129188, Abu Dhabi, UAE \\ ${ }^{6}$ Department of Physics \& Astronomy, University of California, Los Angeles, 430 Portola Plaza, Los Angeles, CA 90095, USA \\ Received 2016 May 10; revised 2016 June 8; accepted 2016 June 8; published 2016 September 8
}

\begin{abstract}
Using a large sample of spectroscopically confirmed $z \sim 3$ galaxies, we establish an empirical relationship between reddening $(E(B-V))$, neutral gas covering fraction $\left(f_{\text {cov }}(\mathrm{H} \mathrm{I})\right)$, and the escape of ionizing (Lyman continuum, LyC) photons. Our sample includes 933 galaxies at $z \sim 3,121$ of which have deep spectroscopic observations $(\gtrsim 7 \mathrm{hr})$ at $850 \lesssim \lambda_{\text {rest }} \lesssim 1300 \AA$ with the Low Resolution Imaging Spectrograph on Keck. The high covering fraction of outflowing optically thick $\mathrm{H}$ I indicated by the composite spectra of these galaxies implies that photoelectric absorption, rather than dust attenuation, dominates the depletion of LyC photons. By modeling the composite spectra as the combination of an unattenuated stellar spectrum including nebular continuum emission with one that is absorbed by $\mathrm{H}$ I and reddened by a line-of-sight extinction, we derive an empirical relationship between $E(B-V)$ and $f_{\text {cov }}(\mathrm{HI})$. Galaxies with redder UV continua have larger covering fractions of $\mathrm{H} \mathrm{I}$ characterized by higher line-of-sight extinctions. We develop a model which connects the ionizing escape fraction with $E(B-V)$, and which may be used to estimate the ionizing escape fraction for an ensemble of galaxies. Alternatively, direct measurements of the escape fraction for our sample allow us to constrain the intrinsic LyC-toUV flux density ratio to be $\langle S(900 \AA) / S(1500 \AA)\rangle_{\text {int }} \gtrsim 0.20$, a value that favors stellar population models that include weaker stellar winds, a flatter initial mass function, and/or binary evolution. Last, we demonstrate how the framework discussed here may be used to assess the pathways by which ionizing radiation escapes from highredshift galaxies.
\end{abstract}

Key words: dark ages, reionization, first stars - dust, extinction - galaxies: evolution - galaxies: formation galaxies: high-redshift - galaxies: ISM

\section{INTRODUCTION}

Significant efforts have focused on determining the sources responsible for cosmic reionization. Studies suggesting the apparent minor role of quasars in keeping the universe ionized at $z \gtrsim 3$ (e.g., Madau et al. 1999; Fan et al. 2001; McDonald \& Miralda-Escudé 2001; Steidel et al. 2001; Bolton et al. 2005; Bolton \& Haehnelt 2007; Siana et al. 2008; Glikman et al. 2011; Fontanot et al. 2012-but see Giallongo et al. 2015; Madau \& Haardt 2015) have led the community to assess the contribution of star-forming galaxies to the ionizing photon budget at high redshift (e.g., Bouwens et al. 2004, 2015; Yan \& Windhorst 2004; Stark et al. 2007; Bunker et al. 2010; McLure et al. 2010; Oesch et al. 2010; Robertson et al. 2010, 2015; Finkelstein et al. 2012; Grazian et al. 2012; Ellis et al. 2013; Oesch et al. 2013; Duncan \& Conselice 2015). These investigations have advocated broadly for the important role of hitherto undetected UV-faint galaxies-with their high number densities as inferred from the steep faint-end slopes of the UV luminosity functions and their assumed large Lyman continuum (LyC) escape fractions-in dominating the ionizing background.

\footnotetext{
* Based on data obtained at the W.M. Keck Observatory, which is operated as a scientific partnership among the California Institute of Technology, the University of California, and NASA, and was made possible by the generous financial support of the W.M. Keck Foundation.

7 Alfred P. Sloan Research Fellow.
}

However, the direct detection of LyC radiation, possible only at modest redshifts $(z \lesssim 3.8)$ where the transmissivity of the inter-galactic medium (IGM) allows such measurements, remains elusive. Locally, only a handful of objects have been detected with LyC emission (Leitet et al. 2013; Borthakur et al. 2014; Izotov et al. 2016a, 2016b; Leitherer et al. 2016). There are a number of statistical detections in stacked spectra (Steidel et al. 2001) and individual detections of candidate LyC emission from both LBGs and Ly $\alpha$-selected samples (Shapley et al. 2006, 2016; Iwata et al. 2009; Vanzella et al. 2010a, 2012; Nestor et al. 2011, 2013; Mostardi et al. 2013; de Barros et al. 2016) at $z \gtrsim 3$. However, foreground contamination has been identified as a significant issue in interpreting the $\mathrm{LyC}$ emission from high-redshift galaxies (Vanzella et al. 2010b, 2012; Nestor et al. 2011, 2013; Mostardi et al. 2015; Siana et al. 2015). Nonetheless, at face value the LyC detections at $z \gtrsim 3$ combined with upper limits on the escape fraction locally and at intermediate redshifts (Leitherer et al. 1995; Hurwitz et al. 1997; Deharveng et al. 2001; Siana et al. 2007, 2010; Cowie et al. 2009; Grimes et al. 2009; Bridge et al. 2010) have motivated an interpretation where the LyC escape fraction-i.e., the fraction of H-ionizing photons that escapes galaxies before being attenuated by either the IGM or the circum-galactic medium (CGM) - evolves toward larger values at higher redshift (Inoue et al. 2006; Siana et al. 2007, 2010; Bridge et al. 2010).

Analyses of the limited number of $\mathrm{LyC}$ detections, as well as observations of the $\operatorname{Ly} \alpha$ escape fractions in high-redshift 
galaxies, suggest that dust reddening and/or gas covering fraction play an important role in modulating the escape of LyC (and Ly $\alpha$ ) photons (e.g., Shapley et al. 2003; Kornei et al. 2010; Hayes et al. 2011; Jones et al. 2013; Rudie et al. 2013; Wofford et al. 2013; Borthakur et al. 2014; Alexandroff et al. 2015; Rivera-Thorsen et al. 2015; Trainor et al. 2015; Dijkstra et al. 2016). Rest-frame UV spectroscopic studies of high-redshift galaxies have focused on the propensity of $\mathrm{Ly} \alpha / \mathrm{LyC}$ escape based on an examination of the gas covering fraction as typically inferred from saturated low-ionization interstellar (IS) absorption lines (e.g., Si II, O I, Fe II) that are relatively straightforward to measure (e.g., Shapley et al. 2003; Jones et al. 2013; Alexandroff et al. 2015; Trainor et al. 2015). However, these transitions may arise in gas of much higher optical depth-e.g., if the ions are cospatial with the densest gas - than that required to significantly attenuate LyC photons $\left(\log \left[N(\mathrm{H} \mathrm{I}) / \mathrm{cm}^{-2}\right] \approx 18\right)$, leading to some ambiguity in the $\mathrm{H}$ I covering fraction. Obviously, one should directly measure the column density and covering fraction of $\mathrm{H}$ I where the $\mathrm{Ly} \alpha / \mathrm{LyC}$ opacity originates. The difficulty with this approach, until recently, has been that: (a) the detection of the $\mathrm{HI}$ absorption lines requires deep far-UV spectroscopy with blue-sensitive spectrographs, and (b) the proper interpretation of these lines requires careful corrections for differential atmospheric refraction relevant at the observed wavelengths, as well as large samples to average over variations in Ly $\alpha$ forest blanketing. Moreover, apart from the anecdotal evidence of a connection between ionizing escape fraction, neutral gas covering fraction, and reddening, a quantitative study of how these properties are related to each other has yet to be undertaken.

To that end, we have used a sample of $z \sim 3$ Lyman Break Galaxies (LBGs) to place constraints on the two mechanisms that act to suppress the escape of ionizing photons: photoelectric absorption by neutral hydrogen (H I) and dust obscuration. In Reddy et al. (2016), subsequently referred to as Paper I, we use our sample of $z \sim 3$ LBGs to deduce the shape of the dust attenuation curve to wavelengths close to the Lyman break. The resulting curve rises less steeply toward bluer wavelengths than other commonly adopted attenuation curves (e.g., Calzetti et al. 2000), implying a factor of $\approx 2$ lower dust attenuation of LyC photons for typical $\left(L^{*}\right)$ galaxies at $z \sim 3$. In this paper, we extend these results by modeling the composite (stacked) UV spectra of $z \sim 3$ galaxies to assess the neutral gas column densities and covering fractions relevant for typical star-forming galaxies at these redshifts. We then draw a connection between reddening, gas covering fraction, and ionizing escape fraction.

The outline of this paper is as follows. In Section 2, we briefly discuss the sample of galaxies and the procedure to construct their far-UV spectral composites. The methodology of fitting models to the composite spectra is presented in Section 3. We discuss the evidence for a non-unity covering fraction of gas in Section 4. The modeling of the stacked UV spectra and the correlations found between continuum reddening and gas covering fraction, column density, and line-of-sight extinction are considered in Section 5. We discuss these correlations in the context of Ly $\alpha$ escape fractions and inferences of gas covering fractions from low-ionization interstellar absorption lines in Section 6. In Section 7, we discuss the implications of our results in the context of the escape of LyC photons from high-redshift galaxies, and how the framework established here can enable powerful constraints on the ionizing stellar populations of highredshift galaxies. All wavelengths are in vacuum. All magnitudes are expressed in the $\mathrm{AB}$ system. We adopt a cosmology with $H_{0}=70 \mathrm{~km} \mathrm{~s}^{-1} \mathrm{Mpc}^{-1}, \Omega_{\Lambda}=0.7$, and $\Omega_{\mathrm{m}}=0.3$.

\section{SAMPLE SELECTION AND REST-FRAME UV SPECTROSCOPY}

The imaging and spectroscopic survey of $z \sim 3$ LBGs presented in Steidel et al. $(2003,2004)$ forms the basis for the sample considered in both Paper I and the present analysis. Here, we briefly recount a few of the salient aspects of our sample, and refer the reader to Paper I for additional details. Candidate $\mathcal{R} \leqslant 25.5$ galaxies were selected to lie at $z \sim 3$ based on their rest-frame UV colors as measured from groundbased $U_{\mathrm{n}} G \mathcal{R}$ imaging of 17 fields. Subsequent shallow spectroscopy of the candidates $(\sim 1.5 \mathrm{hr})$ with the Low Resolution Imaging Spectrograph (LRIS; Oke et al. 1995) on the Keck telescope yielded a sample of 933 star-forming galaxies (AGN were excluded; see PaperI) with secure spectroscopic redshifts in the range $2.00 \leqslant z_{\text {spec }} \leqslant 3.75$ (average redshift of $\left\langle z_{\text {spec }}\right\rangle=2.969$; see Figure 1 of Paper I for the histogram of redshifts). The $z \sim 3 \mathrm{UV}$ luminosity function indicates that galaxies in our sample are typical of those found at these redshifts, with an average luminosity corresponding to $L^{*}$, or an absolute magnitude in the rest-frame UV (1700 ̊) of $M_{1700} \approx-21$ (Steidel et al. 1999; Reddy et al. 2008; Reddy \& Steidel 2009).

Of the 933 galaxies, 121 were targeted with very deep ( $\simeq 7-10 \mathrm{hr}$ ) spectroscopy with the blue arm of LRIS (Steidel et al. 2004), providing rest-frame far-UV coverage at $850 \lesssim \lambda_{\text {rest }} \lesssim 1300 \AA$ (hereafter referred to as the "LyC sample" or the "LyC spectra"). These additional observations were obtained with an atmospheric dispersion corrector to mitigate differential atmospheric refraction most prominent at blue wavelengths. The deep spectroscopy is beneficial not only for the obvious reason of allowing for greater signal-to-noise ratio $(\mathrm{S} / \mathrm{N})$ measurements close to the Lyman Break, but also enables the more efficient identification of LBGs that are contaminated by unresolved foreground objects. The LyC sample excludes a handful of objects identified as spectroscopic blends (see Paper I and Steidel et al., in prep., for further discussion). The rest-frame spectral resolutions of the shallow and deep spectra are $\approx 1.9$ and $1.5 \AA$, respectively. The individual spectra were combined to produce a composite spectrum and associated error spectrum following the procedure presented in Paper I. The $\mathrm{S} / \mathrm{N}$ per resolution element of the final composite spectrum varies from $\mathrm{S} / \mathrm{N} \approx 35$ at $\lambda \simeq 920 \AA$ to $\gtrsim 150$ at $\lambda \simeq 1600 \AA$. The composite of the 933 galaxies in our sample, including the shallow and deep spectra, is shown in Figure 1.

\section{METHODOLOGY}

Our analysis relies on the fitting of spectral models to the composite spectra of $z \sim 3$ galaxies. The composites enable us to average over discrete IGM absorption features in order to accurately model the $\mathrm{H}$ I Lyman absorption lines blueward of Ly $\alpha$. The methodology for fitting models to the stacked spectra is similar to that presented in Paper I. Here, we discuss some basic details of the adopted intrinsic template, the treatment of $\mathrm{H} \mathrm{I}$ and $\mathrm{H}_{2}$ absorption, and the fitting procedures. 

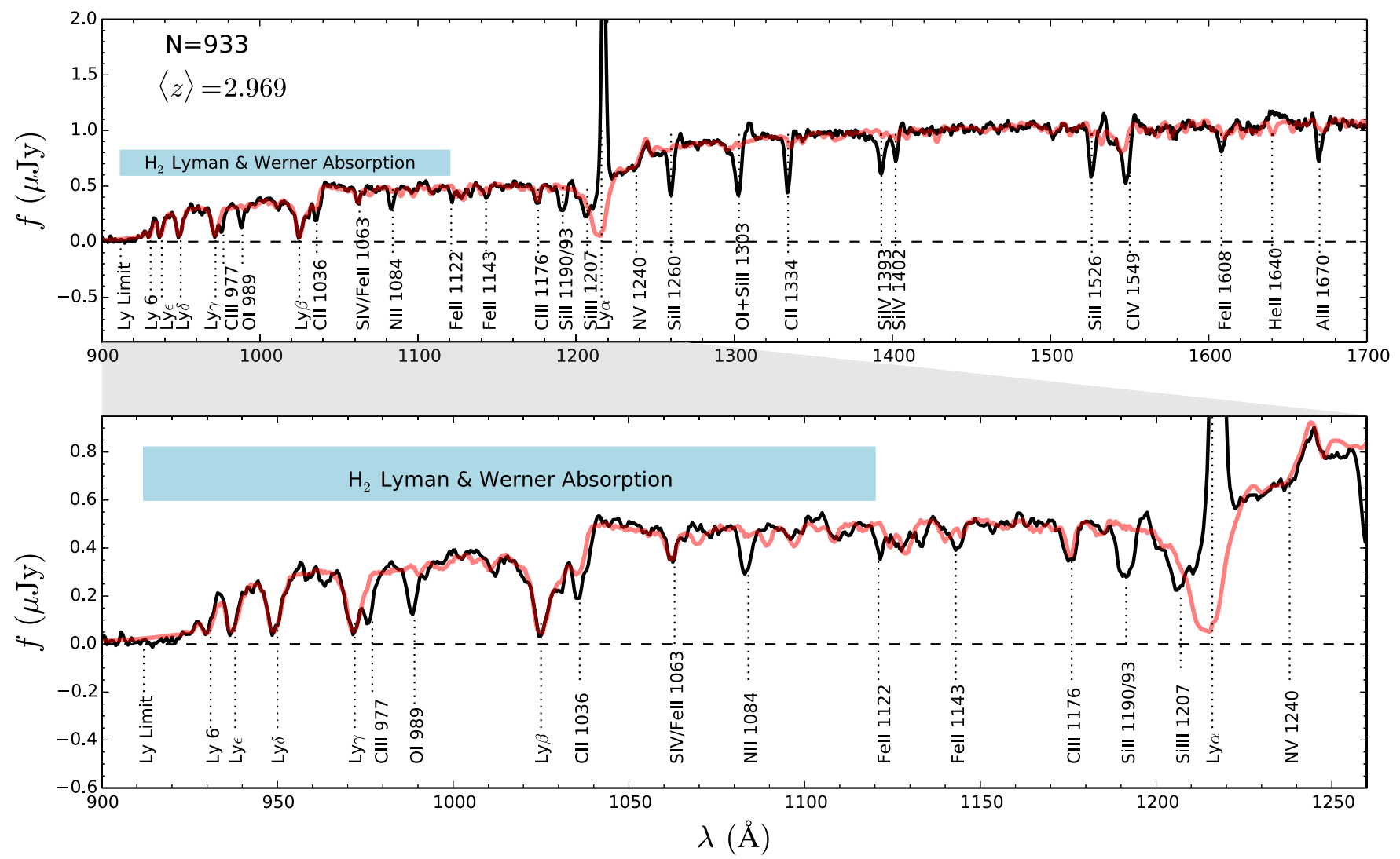

Figure 1. Top: composite spectrum (black) of 933 galaxies at $z \sim 3$ normalized by the median flux density in the range $\lambda=1400-1500 \AA$. Several of the most prominent stellar photospheric and interstellar absorption lines indicated, along with the range of wavelengths $(\lambda=912-1120 \AA)$ corresponding to $\mathrm{H}_{2} \mathrm{Lyman}^{-W e r n e r}$ absorption. The best-fit model to the spectrum (excluding interstellar metal absorption lines) is shown in red. The model assumes an $\mathrm{H} \mathrm{I} /$ dust covering fraction of $f_{\text {cov }}(\mathrm{H} \mathrm{I})=0.96$, a line of sight reddening $E(B-V)_{\text {los }}=0.096$ with an SMC extinction curve, $\log \left[N(\mathrm{H} \mathrm{I}) / \mathrm{cm}^{-2}\right]=21.0$, an $\mathrm{H}_{2}$ covering fraction of $f_{\text {cov }}\left(\mathrm{H}_{2}\right)=0.03$, and $\log \left[N\left(\mathrm{H}_{2}\right) / \mathrm{cm}^{-2}\right]=20.9$. Bottom: same as top panel, zoomed-in on the region between $\lambda=900$ and $1260 \AA$. The composite shows evidence of damping wings in the Lyman series, but line cores that do not reach zero intensity as would be expected for the complete covering of continuum by optically thick H I.

\subsection{Intrinsic Template}

Models were fit to the composite spectra in order to deduce the reddening, average neutral gas column density, and covering fraction for galaxies in our sample. To accomplish this, we constructed an intrinsic template from a combination of the Rix et al. (2004) models with the 2010 version of Starburst99 far-UV spectral synthesis models (Leitherer et al. 1999, 2010). We adopted a stellar metallicity of $0.28 Z_{\odot}$, based on current solar abundances (Asplund et al. 2009), as this metallicity was found to best reproduce the UV stellar wind lines, including C III $\lambda 1176$ and the N V $\lambda 1240$ and C IV $\lambda 1549$ P-Cygni profiles. Furthermore, a constant star formation history and an age of $100 \mathrm{Myr}$ were assumed based on results from stellar population modeling of $z \sim 3$ LBGs presented in Reddy et al. (2012). Nebular continuum emission was added to the stellar template to produce the final "intrinsic template," which we denote by the symbol $m$.

To account for absorption by neutral hydrogen, we added H I Lyman series absorption to the intrinsic template assuming a Voigt profile with a Doppler parameter $b=125 \mathrm{~km} \mathrm{~s}^{-1}$ (the wings of the absorption lines are insensitive to the particular choice of $b$ ) and varying column densities $N(\mathrm{H} \mathrm{I}){ }^{8}$ The $\mathrm{H} \mathrm{I}$

\footnotetext{
8 In Section 5.2, we consider a more realistic line profile for the $\mathrm{H}$ I derived from the composite spectrum itself.
}

absorption was blueshifted by $300 \mathrm{~km} \mathrm{~s}^{-1}$ to account for the observed positions of these lines in the composites. The observed blueshift indicates that most of the neutral hydrogen seen in absorption is outflowing at several hundred $\mathrm{km} \mathrm{s}^{-1}$. Models that include $\mathrm{H}$ I absorption are indicated by the symbol $m(\mathrm{H} \mathrm{I})$. Lastly, we included Lyman-Werner absorption with varying column densities and covering fractions of $\mathrm{H}_{2}$ as described in Paper I. This absorption, which is unresolved, decreases the level of the continuum at $\lambda \simeq 912-1120 \AA$. Models that include both $\mathrm{HI}$ and $\mathrm{H}_{2}$ are referred to by the symbol $m\left(\mathrm{H} \mathrm{I}+\mathrm{H}_{2}\right)$.

\subsection{Spectral Modeling}

We considered two different cases when fitting the stacked spectra. In the first case, we calculated the average reddening of the composite assuming a $100 \%$ covering fraction of dust. This is analogous to deriving the reddening of a galaxy assuming a starburst attenuation curve (e.g., Calzetti et al. 2000; Reddy et al. 2015). In the subsequent discussion, the "updated" versions of the Calzetti et al. (2000) and Reddy et al. (2015) curves refer to those that are updated for our new measurements of the far-UV shape of the dust curve as presented in Paper I. The intrinsic template was reddened by various amounts $(E(B-V)=0.00-0.60)$ assuming the updated attenuation curves. The best-fit $E(B-V)$ was obtained through $\chi^{2}$ minimization by comparing the reddened 
Table 1

Wavelength Windows for Fitting

\begin{tabular}{|c|c|}
\hline Type of Fitting & $\lambda(\AA)$ \\
\hline \multirow[t]{7}{*}{ Composite $E(B-V)^{\mathrm{a}}$} & $\overline{1268-1293}$ \\
\hline & $1311-1330$ \\
\hline & $1337-1385$ \\
\hline & $1406-1521$ \\
\hline & $1535-1543$ \\
\hline & $1556-1602$ \\
\hline & $1617-1633$ \\
\hline \multirow[t]{4}{*}{$N(\mathrm{H} \mathrm{I})^{\mathrm{b}}$} & $967-974$ \\
\hline & $1021-1029$ \\
\hline & $1200-1203$ \\
\hline & $1222-1248$ \\
\hline \multirow[t]{4}{*}{$N\left(\mathrm{H}_{2}\right)^{\mathrm{c}}$} & $941-944$ \\
\hline & $956-960$ \\
\hline & 979-986 \\
\hline & $992-1020$ \\
\hline
\end{tabular}

Notes.

${ }^{\text {a }}$ Windows used to determine the best-fit $E(B-V)$ corresponding to a composite spectrum.

${ }^{\mathrm{b}}$ Windows used to determine the best-fit column density $N(\mathrm{H} \mathrm{I})$ and covering fraction $f_{\text {cov }}(\mathrm{H}$ I) of neutral hydrogen.

${ }^{c}$ Windows used to determine the best-fit column density $N\left(\mathrm{H}_{2}\right)$ and covering fraction $f_{\text {cov }}\left(\mathrm{H}_{2}\right)$ of molecular hydrogen.

templates-i.e.,

$$
m_{\text {final }}=10^{-0.4 E(B-V) k} \times m,
$$

where $k$ is the attenuation curve-to the composite spectrum in the "Composite $E(B-V)$ " wavelength windows listed in Table 1. These windows were chosen to avoid prominent interstellar and stellar absorption/emission features. Note that the windows used to measure $E(B-V)$ are unaffected by $\mathrm{H}$ I or $\mathrm{H}_{2}$ absorption, thus obviating the need to fit for this absorption when determining the reddening of the composite.

In Section 4, we present evidence from the composite spectra that indicates a non-unity covering fraction of gas for galaxies in our sample. In this second case, we fit models to the composite spectra assuming non-unity covering fractions of dust, and neutral and molecular hydrogen. The models were computed as:

$$
\begin{aligned}
m_{\text {final }}= & 10^{-0.4 E(B-V)_{\mathrm{los}} k} \\
& \times\left[\left(f_{\mathrm{cov}}(\mathrm{H} \mathrm{I})-f_{\mathrm{cov}}\left(\mathrm{H}_{2}\right)\right) m(\mathrm{H} \mathrm{I})\right. \\
& \left.+f_{\text {cov }}\left(\mathrm{H}_{2}\right) m\left(\mathrm{H} \mathrm{I}+\mathrm{H}_{2}\right)\right] \\
& +\left(1-f_{\mathrm{cov}}(\mathrm{H} \mathrm{I})\right) m .
\end{aligned}
$$

Here, $f_{\text {cov }}(\mathrm{H} \mathrm{I})$ is the covering fraction of dust and neutral gas, and $f_{\text {cov }}\left(\mathrm{H}_{2}\right)$ is the covering fraction of $\mathrm{H}_{2}$. This equation assumes that the $\mathrm{H}_{2}$ is shielded by $\mathrm{H}$ I and that only the portion of the spectrum that emerges through the $\mathrm{HI}$ and $\mathrm{H}_{2}$ gas is reddened. As shown in Sections 4.3 and 5, this particular formalism is motivated by the fact that the ISM is expected to be porous and that the covering fraction of $\mathrm{H}_{2}$ is much smaller than that of $\mathrm{HI}$ (see Paper I and below). The last term in Equation (2) corresponds to the portion of the light that exits the galaxy unabsorbed, which is simply the intrinsic template multiplied by $1-f_{\text {cov }}(\mathrm{H} \mathrm{I})$. Finally, $k$ in this equation now refers to a line-of-sight extinction curve in lieu of an attenuation curve, and $E(B-V)_{\text {los }}$ refers to the corresponding line-of-sight reddening. These adjustments assume that a foreground uniform dust screen describes the dust-covered portions of the galaxy.

The models were computed assuming a range of line-of-sight reddening, and covering fractions and column densities of $\mathrm{H} \mathrm{I}$ and $\mathrm{H}_{2}$. In all cases, the models were smoothed to the spectral resolution of the composites, and modified by the CGM and IGM opacity appropriate for the average redshift of each composite. The average opacity was determined by randomly drawing line-of-sight absorber column densities from the distribution measured in the Keck Baryonic Structure Survey (Rudie et al. 2013), and taking an average over many lines-ofsight. ${ }^{9}$ The best-fitting $E(B-V)_{\text {los }}, f_{\text {cov }}(\mathrm{H} \mathrm{I})$, and $N(\mathrm{H} \mathrm{I})$ were found by comparing the models to the stacked spectra in the "Composite $E(B-V)$ " and "N(H I)" windows listed in Table 1. As discussed in Paper I, the " $N(\mathrm{H} \mathrm{I})$ " windows were chosen to include the core and the wings of $\operatorname{Ly} \beta$ and $\mathrm{Ly} \gamma$, and the wings of $\operatorname{Ly} \alpha$, while excluding regions of metal line absorption (e.g., $\mathrm{C}$ III $\lambda 977$ and $\mathrm{C}$ II $\lambda 1036$ ). The region between $\operatorname{Ly} \alpha$ and $\mathrm{NV} \lambda 1238$ is particularly sensitive to the gas column density and is included in the fitting windows. The higher order Lyman transitions (e.g., Ly $\delta$, Ly $\epsilon$, etc.) were not used in the fitting due to line overcrowding. Furthermore, as noted above, the fitting windows do not include any interstellar metal absorption lines. Similarly, the best-fit values of $f_{\text {cov }}\left(\mathrm{H}_{2}\right)$ and $N\left(\mathrm{H}_{2}\right)$ were determined by comparing the models to the stacked spectra in the " $N\left(\mathrm{H}_{2}\right)$ " windows listed in Table 1. As discussed in Paper I, the "N($\left(\mathrm{H}_{2}\right)$ " windows were chosen to avoid regions of strong metal line and $\mathrm{H}$ I absorption.

\section{NON-UNITY COVERING FRACTION OF GAS IN TYPICAL GALAXIES AT $z \sim 3$}

The apparent porosity of the ISM in galaxies-examples of which have been seen or suggested in the local universe (e.g., Silk 1997; Kunth et al. 1998; Clarke \& Oey 2002; Oey et al. 2002; Bagetakos et al. 2011; Heckman et al. 2011), and which may be enhanced by the high star formation rate (SFR) surface densities characteristic of high-redshift galaxies (e.g., Razoumov \& Sommer-Larsen 2010)—motivates spectral models that allow for a less than complete covering of the continuum by dust and neutral gas. Notably, the composite spectrum (Figure 1) exhibits $\mathrm{H} \mathrm{I}$ absorption lines that have damping wings, but which do not reach zero intensity at the line cores. The residual flux density under the $\mathrm{H}$ I lines is $\approx 5 \%$ of the median flux density in the range $\lambda=1400-1500 \AA$. For the normalization shown in Figure 1, the residual flux density is $\simeq 0.05 \mu \mathrm{Jy}$. As shown in Paper I, the H I damping wings are best reproduced by high column density gas with $\log \left[N(\mathrm{H} \mathrm{I}) / \mathrm{cm}^{-2}\right] \approx 21.0$. At face value, these results suggest a partial covering fraction of high column density gas. However, we sought first to rule out other possible sources for the residual flux observed at the $\mathrm{H}$ I line cores, namely

\footnotetext{
Adopting a different prescription for the IGM opacity (e.g., O'Meara et al. 2013; Inoue et al. 2014) does not affect the derived H I covering fractions as these fractions are sensitive to the ratio of the line depth to the continuum, both of which will be affected equally by any changes in the IGM opacity. However, because the $\mathrm{H}_{2}$ covering fraction is calculated by "matching" the model continuum to that of the composite spectrum in the $\mathrm{H}_{2}$ windows (Table 1), this quantity will suffer a systematic bias related to our choice of the IGM+CGM opacity prescription (see below).
} 
uncertainties in redshifts and spectral resolution, and foreground contamination.

\subsection{Redshift and Spectral Resolution Uncertainties}

We determined the extent to which uncertainties in the redshifts and the resolution of the individual galaxy spectra lead to non-zero fluxes in the H I line cores in the stacked spectrum. To accomplish this, we added $\mathrm{HI}$ absorption of varying column densities $\log \left[N(\mathrm{H} \mathrm{I}) / \mathrm{cm}^{-2}\right]=20.0-22.0$ to the intrinsic template, assuming (as above) a Voigt profile with a Doppler parameter $b=125 \mathrm{~km} \mathrm{~s}^{-1}$, and a $100 \%$ covering of gas, resulting in an "H I-absorbed template." Thus, this $\mathrm{H}$ I-absorbed template has saturated black $\mathrm{H}$ I line cores. The $\mathrm{H}$ I-absorbed template was then smoothed to have spectral resolutions varying from $1.5 \AA$ (the average rest-frame resolution inferred for the $\mathrm{LyC}$ spectra) up to a factor of two times worse $(3.0 \AA)$. For each value of the spectral resolution, we generated 121 versions of the smoothed $\mathrm{H}$ I-absorbed templates - one for each of the $121 \mathrm{LyC}$ spectra contributing to the composite at $\lambda<1150 \AA$-where each version was shifted in wavelength according to a normal distribution with $\sigma=125 \mathrm{~km} \mathrm{~s}^{-1}$. This velocity spread is based on the uncertainties in the systemic redshifts when they are derived using interstellar absorption lines (see Steidel et al. 2010), and the vast majority of the LyC spectra have systemic redshifts based on the latter. These 121 versions were then averaged together to produce a composite model. Based on these simulations, we would have had to systematically underestimate the average resolution of the LyC spectra by $\gtrsim 50 \%$ in order to match the $\mathrm{HI}$ line depths for $\log \left[N(\mathrm{H} \mathrm{I}) / \mathrm{cm}^{-2}\right]>20.0$, a highly unlikely situation given that this $50 \%$ systematic difference is much larger than the rms uncertainty $(<0.1 \AA)$ in determining the spectral resolution from the widths of the night sky lines. For the actual value of the resolution of our spectra and our typical redshift uncertainty, the cores of the $\mathrm{H}$ I lines remain black, implying that none of the observed residual flux density can be attributed to spectral resolution or redshift uncertainties. We conclude that systematic uncertainties in spectral resolution cannot account for the residual line flux density at the $\mathrm{HI}$ line cores.

\subsection{Foreground Contamination}

We ruled out foreground contamination-i.e., low-redshift objects that are unresolved from the LBGs in the ground-based photometry and spectroscopy-as a possible origin for the residual line flux observed at the $\mathrm{HI}$ line cores based on multiple lines of reasoning. First, we examined the contamination statistics for the sample of LBGs with spatially resolved Hubble Space Telescope (HST) multi-filter (UVJH) and ground-based NB3420 imaging (to target $\mathrm{LyC}$ emission at $z \sim 3)$ analyzed in Mostardi et al. $(2013,2015)$. Their sample contains 16 LBGs with HST imaging, four of which have NB3420 detections. Of these four, two were identified as having foreground contaminants, such that the offending lowredshift interlopers would not have been resolved separately from the background LBGs in the ground-based photometry. Of the 12 objects that were not detected in the NB3420 imaging, only one suffers from foreground contamination. Thus, the contamination rates for NB3420 detections and nondetections are $2 / 4$ and $1 / 12$, respectively. Extrapolating to the full LBG sample of Mostardi et al. (2013), where five of 49 objects were detected in the narrow-band imaging, implies that the fraction of LBGs with contaminated photometry is $2 / 4 \times 5 / 49+1 / 12 \times 44 / 49 \approx 0.13$. Note that what we have just computed is the foreground contamination rate. However, the quantity of relevance in the present context is not the contamination rate, but the actual fraction of flux contributed by foreground contaminants.

To calculate this flux contamination, we examined the photometry for the 16 LBGs with $H S T$ imaging from Mostardi et al. (2015). We summed the UVJH flux densities for the 16 objects, both including and excluding foreground contaminants that would have been unresolved from the LBGs in the groundbased imaging. The ratio of the total flux in each band including foreground contamination to that excluding this contamination is $2.38,1.02,1.03$, and 1.02 , respectively, for the $U V J H$ bands. Accounting for the higher foreground contamination rate among the 16 LBGs $(3 / 16 \approx 0.19)$ relative to the rate among the full LBG sample ( $\approx 0.13$, see above), and adopting the reasonable assumption that the flux contamination per object is roughly constant, the aforementioned ratios become $1.92,1.01,1.02$, and 1.01, respectively, for the $U V J H$ bands. In other words, the flux contamination fraction for the rest-frame non-ionizing UV/optical flux is $<2 \%$. The flux contamination fraction in the $U$-band is substantially larger, a point that we revisit in Section 7. Based on these statistics, the contaminating flux density across the wavelength range spanning the $\operatorname{Ly} \beta, \operatorname{Ly} \gamma$, and $\operatorname{Ly} \delta$ lines is a factor of $\approx 5$ lower than the residual flux densities measured at the aforementioned line centers.

Note that the previously discussed contamination rates were calculated for LBGs that were spectroscopically confirmed using relatively shallow spectroscopic data (Reddy et al. 2008; Steidel et al. 2011; Mostardi et al. 2013). Spectroscopic blends may not be as readily identifiable in these shallow spectra compared to the substantially deeper LyC spectra analyzed here. As indicated in Section 2, the $\simeq 7-10 \mathrm{hr}$ spectroscopic integrations obtained with LRIS-B allowed us to remove a handful $(\approx 5 \%)$ of LBGs that were confused with foreground interlopers. In fact, this contaminating rate is consistent with the expected number of chance superpositions of foreground objects, as discussed in C. C. Steidel et al. (2016, in preparation), implying that the present sample has been expunged of most contaminants. Thus, the contamination rates calculated above should be regarded as conservative upper limits.

There is additional evidence that suggests that the residual line flux at the $\mathrm{H}$ I line centers is intrinsic to the LBGs. Namely, in the subsequent analysis, we show that this residual flux varies systematically with $E(B-V)$ such that redder galaxies exhibit lower levels of residual emission (Section 5), a trend that would not be expected if foreground interlopers were dominating the observed flux. For example, of the three LBGs identified with foreground contamination from the sample of Mostardi et al. (2015), two have interlopers that result in slightly redder colors compared to the corrected photometry, and one has an interloper that results in a color that is indistinguishable from the corrected color. Notwithstanding the small statistics, it follows that an increase in contamination fraction for galaxies with redder $E(B-V)$ should lead to a corresponding increase in residual flux, a trend that is opposite to the one actually observed (Section 5). 


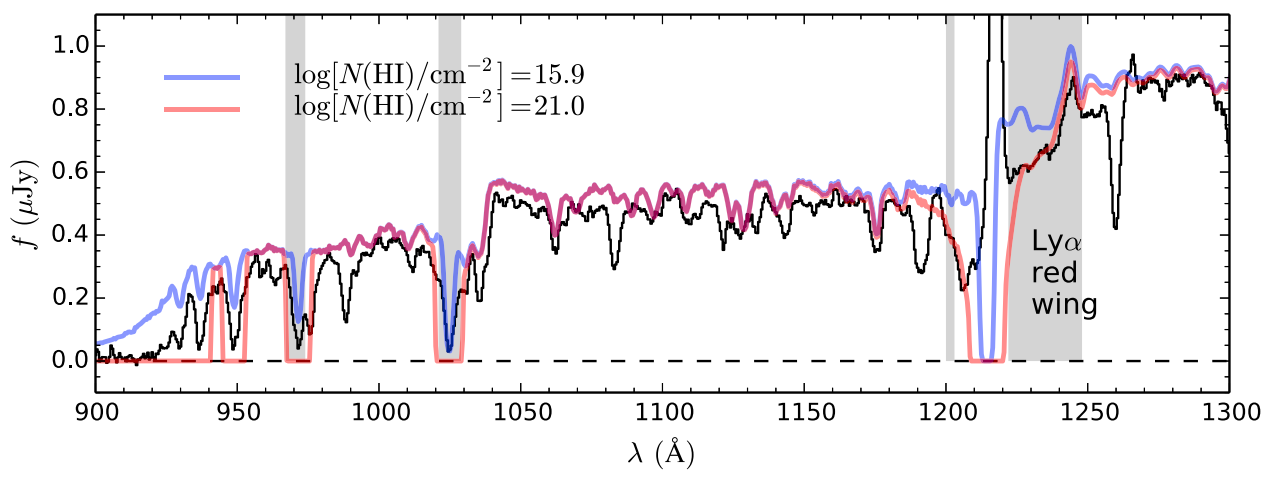

Figure 2. Model fits to the $E(B-V)$ and $\mathrm{H} \mathrm{I}$ lines-assuming $100 \%$ covering of dust and neutral gas with two different column densities, and assuming that the $\mathrm{H} \mathrm{I}$ lines are described by a Voigt profile - to the composite spectrum of all galaxies in our sample (Figure 1). The models have been smoothed to the same spectral resolution as the composite spectra. For reference, the four windows used to fit the Lyman series lines are indicated by the shaded regions (Table 1). The windows around Ly $\alpha$ are designed to exclude the regions with strong Ly $\alpha$ emission, Si III $\lambda 1207$ absorption, and include the red wing of Ly $\alpha$. A low column density of $\log \left[N(\mathrm{H} \mathrm{I}) / \mathrm{cm}^{-2}\right]=15.9$ matches the depth of the $\mathrm{Ly} \beta$ line, but fails to reproduce the red damping wing of Ly $\alpha$ (which is blended with the N v P-Cygni absorption) and the depths of the higher Lyman series lines. A high column density of $\log \left[N(\mathrm{H} \mathrm{I}) / \mathrm{cm}^{-2}\right]=21.0$ is able to reproduce the red damping wing of Ly $\alpha$, but implies saturated H I lines. The roughly equal depths of the Lyman series lines with residual intensity at the line cores implies a partial covering fraction of high column density neutral gas.

\subsection{Summary of the Evidence for a Non-unity Covering Fraction of $\mathrm{H} I$}

To summarize our arguments, we find that systematic uncertainties in the resolution of our spectra, combined with random uncertainties in systemic redshifts, cannot explain the level of residual flux seen in the stacked spectra. Furthermore, calculations of the flux contamination fraction for the sample of LBGs examined in Mostardi et al. $(2013,2015)$ imply that $>80 \%$ of the residual flux must be intrinsic to the LBGs and not a result of foreground interlopers. If the statistics of how the colors of LBGs are altered due to foreground interlopers extend to larger samples, then significant contamination would be expected to lead to a higher residual flux for redder galaxies, which is contrary to the trend that we observe, as discussed in Section 5 .

We conclude with some words of caution regarding the covering fractions derived here. First, while the composite may indicate $f_{\text {cov }}(\mathrm{H} \mathrm{I})<1$, low resolution spectroscopy may mask the presence of very narrow absorption components that are opaque to LyC radiation. ${ }^{10}$ However, if the presence of such narrow (and black) components were the rule rather than the exception for galaxies in our sample, we would expect little correlation between the covering fraction derived from low resolution spectra and the Ly $\alpha$ escape fraction, counter to our observations (Sections 5 and 6).

Second, the method for computing $f_{\text {cov }}(\mathrm{H}$ I) assumes that the different velocity components of the $\mathrm{HI}$ gas are spatially coincident when viewed in projection. If this is not the casei.e., if optically thick gas that absorbs far from line center covers sightlines that are disjoint from those where the absorption at line center dominates-then $f_{\text {cov }}(\mathrm{H} \mathrm{I})$ deduced based on the overall depth of the H I absorption will underestimate the true $\mathrm{H}$ I covering fraction. As we show in Section 7, the two effects just mentioned (i.e., unresolved narrow absorption and spatially disjoint gas at different velocities) must be limited in scope. Even so, in light of this discussion, one must consider that the covering fractions

\footnotetext{
10 These narrow absorption components cannot have column densities much larger than the ones deduced from the composite spectrum, otherwise their presence would be revealed by substantially broader Lorentzian wings.
}

derived in our subsequent analysis may be lower limits to the true fractions.

To demonstrate the requirement of a non-unity covering fraction, we modeled the composite spectrum of all galaxies in our sample (Figure 1) using the same procedure as above in fitting $E(B-V)$ and $\mathrm{H}$ I (we ignored $\mathrm{H}_{2}$ for this exercise), but where we now assumed a unity covering fraction of both dust and gas. The depth of the $\operatorname{Ly} \beta$ line can be reproduced with $\log \left[N(\mathrm{H} \mathrm{I}) / \mathrm{cm}^{-2}\right]=15.9$, but such a low column density does not match the combined red damping wing of $\operatorname{Ly} \alpha$ and P-Cygni N V absorption, nor the depths of the higher Lyman series lines (Figure 2). Alternatively, the assumption of $\log \left[N(\mathrm{H} \mathrm{I}) / \mathrm{cm}^{-2}\right]=21.0$ gas reproduces the combined red damping wing of Ly $\alpha$ and P-Cygni $\mathrm{N} \mathrm{V}$ absorption, but implies optically thick gas and hence saturated $\mathrm{H}$ I lines that should reach zero intensity at the line cores for a unity covering fraction.

Thus, it is clear that neither low or high column density gas with complete covering can simultaneously reproduce the residual flux at the $\mathrm{H}$ I line centers and the damping wings of Ly $\alpha$. Rather, the roughly equal depths of the Lyman series lines combined with the damping wings of $\operatorname{Ly} \alpha$ suggest optically thick gas with $\log \left[N(\mathrm{H} \mathrm{I}) / \mathrm{cm}^{-2}\right] \gtrsim 21.0$ and $f_{\text {cov }}(\mathrm{H} \mathrm{I})<1$. Consequently, the depths of the $\mathrm{H}$ I lines and the red damping wing of Ly $\alpha$ are most sensitive to changes in the covering fraction of optically thick $\mathrm{H} \mathrm{I}$.

\subsection{A Physical Picture for a Non-unity Covering Fraction of Gas and Dust}

From a physical standpoint, a partial covering of dust and gas can result from supernovae chimneys or galactic fountains, whereby supernovae explosions heat the ISM and drive hot gas into the galactic halo (e.g., Putman et al. 2012 and references therein). Aside from direct observations of such hot halos in the Milky Way and other nearby galaxies (e.g., Lynds \& Sandage 1963; Shapiro \& Field 1976; Armus et al. 1995; Lehnert \& Heckman 1995; Strickland et al. 2004), the higher SFR surface densities of - and the ubiquitous presence of outflows in-high-redshift galaxies may increase the porosity of the ISM. Furthermore, simulations of high-redshift galaxies show that the LyC escape fraction is essentially independent of 


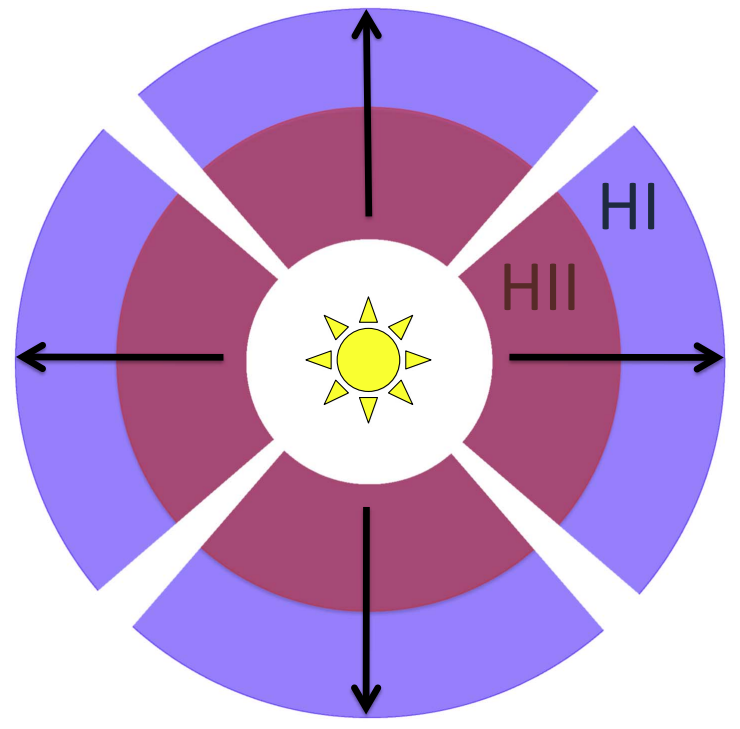

Figure 3. Illustrated configuration of a galaxy with a porous ISM and hence non-unity covering fraction of dust and gas. In this geometry, the ionized region is bounded by neutral gas (i.e., corresponding to a radiation-bounded nebula), and both the ionized and neutral components are outflowing, as indicated by the arrows. Note that the composite spectrum (Figure 1) also indicates non-negligible $\mathrm{H}$ I opacity at systemic redshift. Holes in the ISM, such as those formed by supernovae, result in regions of very low gas and dust column density. In this framework, the dust is coincident with the gas, but may be inhomogeneously distributed between the neutral and ionized phases.

dust obscuration, with the ionizing photons escaping the galaxies through channels that are essentially free of dust (Gnedin et al. 2008; Razoumov \& Sommer-Larsen 2010; Ma et al. 2016), as depicted in Figure 3. In this illustration, the column of gas is sufficient to completely bound the ionized region by neutral gas, where the bulk of the neutral and ionized gas along the line of sight are entrained in an outflow (see also Figure 1 in Zackrisson et al. 2013).

\section{SPECTRAL FITTING RESULTS}

\subsection{An Initial Fit to the Full Composite Spectrum}

Using the procedure laid out in Section 3, we fit a spectral model to the composite of all galaxies (full composite), assuming a non-unity covering fraction of dust and gas, and an SMC extinction curve. The model was obtained by simultaneously varying $E(B-V)_{\text {los } / \mathrm{SMC}}, f_{\text {cov }}(\mathrm{H} \mathrm{I}), N(\mathrm{H} \mathrm{I}), f_{\text {cov }}\left(\mathrm{H}_{2}\right)$, and $N\left(\mathrm{H}_{2}\right)$, until a best-fit was reached. The best-fit parameters are $E(B-V)_{\operatorname{los} / \mathrm{SMC}}=0.096, \quad \log \left[N(\mathrm{H} \mathrm{I}) / \mathrm{cm}^{-2}\right]=21.0$, $f_{\text {cov }}(\mathrm{H} \mathrm{I})=0.96, \quad \log \left[N\left(\mathrm{H}_{2}\right) / \mathrm{cm}^{-2}\right]=20.9, \quad$ and $f_{\text {cov }}\left(\mathrm{H}_{2}\right)=0.03$. By perturbing the composite spectrum by its errors and refitting these realizations, we estimated the errors in $E(B-V)_{\operatorname{los}}, f_{\text {cov }}(\mathrm{H} \mathrm{I})$, and $\log \left[N(\mathrm{H} \mathrm{I}) / \mathrm{cm}^{-2}\right]$ to be $\sigma\left(E(B-V)_{\mathrm{los}}\right) \approx 0.01, \quad \sigma\left(f_{\mathrm{cov}}(\mathrm{H} \mathrm{I})\right) \approx 0.01$, and $\sigma\left(\log \left[N(\mathrm{H} \mathrm{I}) / \mathrm{cm}^{-2}\right]\right) \approx 0.20 \mathrm{dex}$, respectively. Nominally, these errors include the covariance between the parameters. However, as $f_{\text {cov }}(\mathrm{H} \mathrm{I})$ is determined primarily by the depth of the $\mathrm{H}$ I lines and $N(\mathrm{H} \mathrm{I})$ is constrained primarily by the wings, there is negligible covariance between the covering fraction and column density of H I. Furthermore, as noted in Paper I, the $\mathrm{H}_{2}$ lines are unresolved and therefore we cannot accurately determine the errors in the $\mathrm{H}_{2}$ covering fraction and column density. In other words, for the models to match the continuum level of the stacked spectrum in the " $N\left(\mathrm{H}_{2}\right)$ " windows, the $\mathrm{H}_{2}$ column density may be increased while the covering fraction is decreased, or vice versa. As noted above, $f_{\text {cov }}\left(\mathrm{H}_{2}\right)$ may also suffer a systematic bias depending on which IGM opacity prescription we adopt.

\subsection{Derivation of the $H$ I Line Profile}

As shown in Figure 2 and in Paper I, a Voigt profile provides a poor description for the shape of the $\mathrm{H}$ I lines (i.e., the Voigt profile underestimates the width of the lines when simultaneously matching their depths). To obtain a better fit for these lines, we derived the shape of the $\mathrm{H}$ I line profile from the composite spectrum itself. To recover the $\mathrm{H}$ I line profile, the intrinsic spectrum was multiplied by $1-f_{\text {cov }}(\mathrm{H} \mathrm{I})$, modified for the opacity of the IGM, and subtracted from both the composite and its model fit, with the resulting spectra shown in Figure 4. This step allows us to remove the contribution of unabsorbed continuum prior to modeling the $\mathrm{H}$ I lines. We then used the model fit subtracted by the intrinsic spectrum (i.e., red curve in Figure 4) to define "continuum" points by which the $\operatorname{Ly} \beta, \operatorname{Ly} \gamma$, and $\operatorname{Ly} \delta$ line profiles were normalized. There is a single continuum point each for $\operatorname{Ly} \beta$ and $\operatorname{Ly} \gamma$ and we treated the continuum as constant across these line profiles. For Ly $\delta$, the continuum was estimated as a linear function passing through the two continuum points bracketing the line. The normalized line profiles, transformed into velocity space (where the minimum of each absorption trough is forced to zero velocity), and cast in terms of the optical depth, are shown in Figure 4.

The optical depth profile shown in Figure 4 was assumed for all of the Lyman series lines, except Ly $\alpha$. Because the damping wings are weaker and more difficult to measure for the higher Lyman series lines due to line crowding, we modeled the damping wings of $\mathrm{Ly} \alpha$ using a range of $\mathrm{H}$ I column densities with a Doppler parameter $b=125 \mathrm{~km} \mathrm{~s}^{-1}$ —as noted earlier, the wings of the absorption are insensitive to the particular choice of $b$.

\subsection{Composite Fitting}

In order to investigate trends between continuum color excess and covering fraction, we divided the galaxies in our sample into five bins of $E(B-V)$. Specifically, the intrinsic template was reddened with different values of $E(B-V)$ assuming the Reddy et al. (2015) attenuation curve and a $100 \%$ covering fraction of dust, shifted to the observed frame for the redshift of each galaxy, multiplied by the IGM opacity relevant for that redshift, and then convolved with the $G$ and $\mathcal{R}$ filters. The best-fit $E(B-V)$ was taken to be the value that resulted in a model-generated $G-\mathcal{R}$ that best matched the observed $G-\mathcal{R}$ color, where the latter was corrected for Ly $\alpha$ emission or absorption as measured from the spectra. Composite spectra for objects in each bin were constructed according to the procedures laid out in Paper I and Section 2.

In fitting the stacked spectra assuming a porous ISM, we considered both the SMC and Milky Way extinction curves in the modeling (i.e., for the line-of-sight reddening) to span the possible shapes of the extinction curve in both low and high metallicity environments. The fitting was accomplished by simultaneously varying $E(B-V)_{\text {los }}, N(\mathrm{H} \mathrm{I})$ (which affects the degree of damping of the Ly $\alpha$ line), $f_{\text {cov }}(\mathrm{H} \mathrm{I})$ (which affects the depths of all the Lyman series lines), and $f_{\text {cov }}\left(\mathrm{H}_{2}\right)$, until the differences between the composite spectra and the models were 

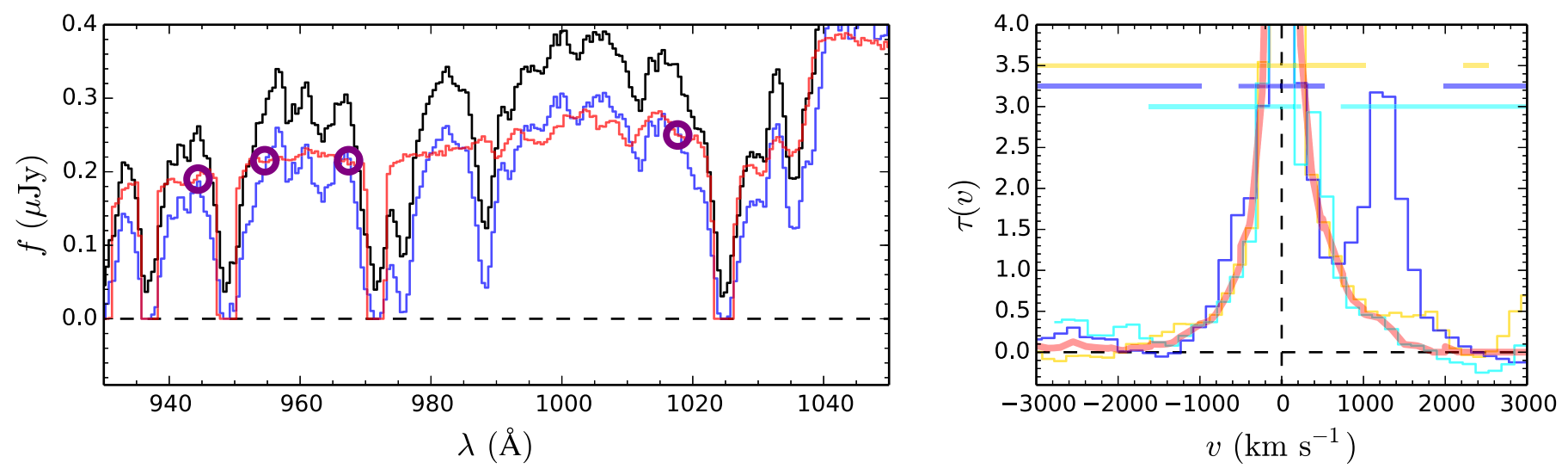

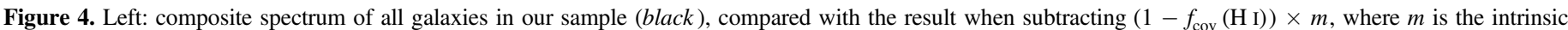

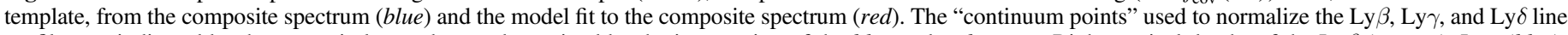

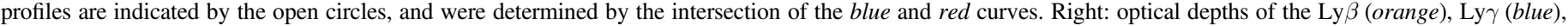

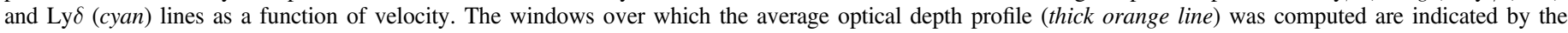
horizontal yellow, blue, and cyan lines for the $\operatorname{Ly} \beta, \operatorname{Ly} \gamma$, and $\operatorname{Ly} \delta$ lines, respectively.

minimized in the wavelength regions indicated by the "Composite $E(B-V)$," "N(H I)," and " $N\left(\mathrm{H}_{2}\right)$ " entries in Table 1. As the $\mathrm{H}_{2}$ lines are unresolved, we fixed $\log \left[N\left(\mathrm{H}_{2}\right) / \mathrm{cm}^{-2}\right]=20.9$ in the fitting. We verified that the covering fractions of neutral gas obtained with and without including $\mathrm{H}_{2}$ in the model fits were similar within $\approx 1 \%$. The covering fractions of $\mathrm{H}_{2}$ are significantly smaller, with a typical value of $f_{\text {cov }}\left(\mathrm{H}_{2}\right) \lesssim 0.05$. Additionally, each composite spectrum was fit assuming a $100 \%$ covering fraction of dust in order to derive its $E(B-V)$. The $E(B-V)$ computed from the composite spectra agree well with the average $E(B-V)$ of objects contributing to those spectra. For simplicity, in the following discussion the color excess derived assuming a unity covering fraction of dust is referred to as the "continuum reddening," $E(B-V)$, while the reddening deduced assuming a partial covering fraction of dust is referred to as the "line-of-sight reddening," $E(B-V)_{\text {los. }}$. The continuum reddenings derived assuming the updated attenuation curves of Reddy et al. (2015) and Calzetti et al. (2000) are denoted by " $E(B-V)_{\text {MOS" }}$ " and " $E(B-V)_{\text {Calz }}$," respectively.

The fits assuming the SMC extinction curve and a non-unity covering fraction of dust and neutral gas are shown in Figure 5, and the best-fit parameters and their errors are summarized in Table 2. The errors were calculated as the $1 \sigma$ dispersion in the values obtained by perturbing the composite spectra according to their error spectra and re-fitting these realizations. Not surprisingly, the new $\mathrm{H}$ I optical depth profile adopted for $\mathrm{Ly} \beta$ and the higher Lyman series transitions (Section 5.2) provides a significantly better fit for these lines-as well as the continuum level at $\lambda<950 \AA$ - than a single Voigt profile, though the inferred covering fractions remain identical (see Figure 1 for the model fit to the full composite).

\subsection{Summary of the Spectral Fitting Results}

Several notable results from the fitting are summarized in Figure 6 and Table 3. Significant correlations are found between the continuum reddening and the line-of-sight reddening, the column density of $\mathrm{H} \mathrm{I}$, and the covering fraction of H I. For simplicity in the subsequent analysis, and unless indicated otherwise, we assume the updated Reddy et al. (2015) curve for the continuum reddening and the SMC extinction curve for deriving the line-of-sight extinction and the neutral gas covering fraction and column density. Other combinations of the extinction/attenuation curves yield similar results to those obtained with the default choices specified above.

To compute the significance of the correlations between $E(B-V)$ and $E(B-V)_{\text {los }}, \quad N(\mathrm{H} \mathrm{I})$, and $f_{\text {cov }}(\mathrm{H} \mathrm{I})$, we calculated the Pearson correlation coefficient and probability of a null correlation between the various quantities while perturbing the measured values many times according to their measurement uncertainties. The values reported in Table 3 are the median Pearson correlation coefficients and median probabilities of a null correlation between $E(B-V)$ and $f_{\text {cov }}(\mathrm{HI}), N(\mathrm{H} \mathrm{I})$, and $E(B-V)_{\text {los. }}$ The inferred $\mathrm{H}$ I covering fractions assuming a partial covering fraction of both dust and gas are $\gtrsim 92 \%$, substantially larger than those deduced assuming a unity covering fraction of dust $\left(f_{\text {cov }}(\mathrm{H} \mathrm{I}) \simeq 0.70-0.80\right.$; see Paper I). In the latter case, as the entire spectrum is reddened including the uncovered portion, the line depths can be reproduced with smaller covering fractions of $\mathrm{H} \mathrm{I}$. With a non-unity covering fraction of dust, larger $\mathrm{H}$ I covering fractions are required to reproduce the depths when unreddened continuum is contributed from the uncovered portion of the galaxy spectrum. At any rate, our results imply that the reddening of the UV continuum slope reflects an increasing covering fraction of $\mathrm{H} \mathrm{I}$ and dust, where the covering gas is also characterized by a higher line-of-sight reddening.

Furthermore, the column density of $\mathrm{H} \mathrm{I}$ increases with continuum reddening. The ratio of the neutral gas column density and the line-of-sight reddening is only marginally correlated with continuum reddening with a Pearson correlation coefficient of $\rho=0.56$ and a probability of null correlation of $p=0.28$ (Figure 7). The mean ratio of the neutral gas column density and the line-of-sight reddening for our sample is $\left\langle N(\mathrm{H} \mathrm{I}) / E(B-V)_{\text {los }}\right\rangle=(5.8 \pm 3.3) \times 10^{21}$ and $(5.4 \pm 3.7) \times 10^{21} \mathrm{~cm}^{-2} \mathrm{mag}^{-1}$ for the SMC and Milky Way extinction curves, respectively, where the errors represent the standard deviation in the five values contributing to the mean. For context, the average values found for SMC and MW sightlines are $\sim 2.1 \times 10^{22}$ and $\sim 4.5 \times 10^{21} \mathrm{~cm}^{-2} \mathrm{mag}^{-1}$, respectively (Welty et al. 2012). A note of caution is that the $\left\langle N(\mathrm{H} \mathrm{I}) / E(B-V)_{\text {los }}\right\rangle$ values calculated here are analogous to, but not the same as, the gas-to-dust ratio. Specifically, we do not make any accounting of either the molecular or ionized gas in our column density 


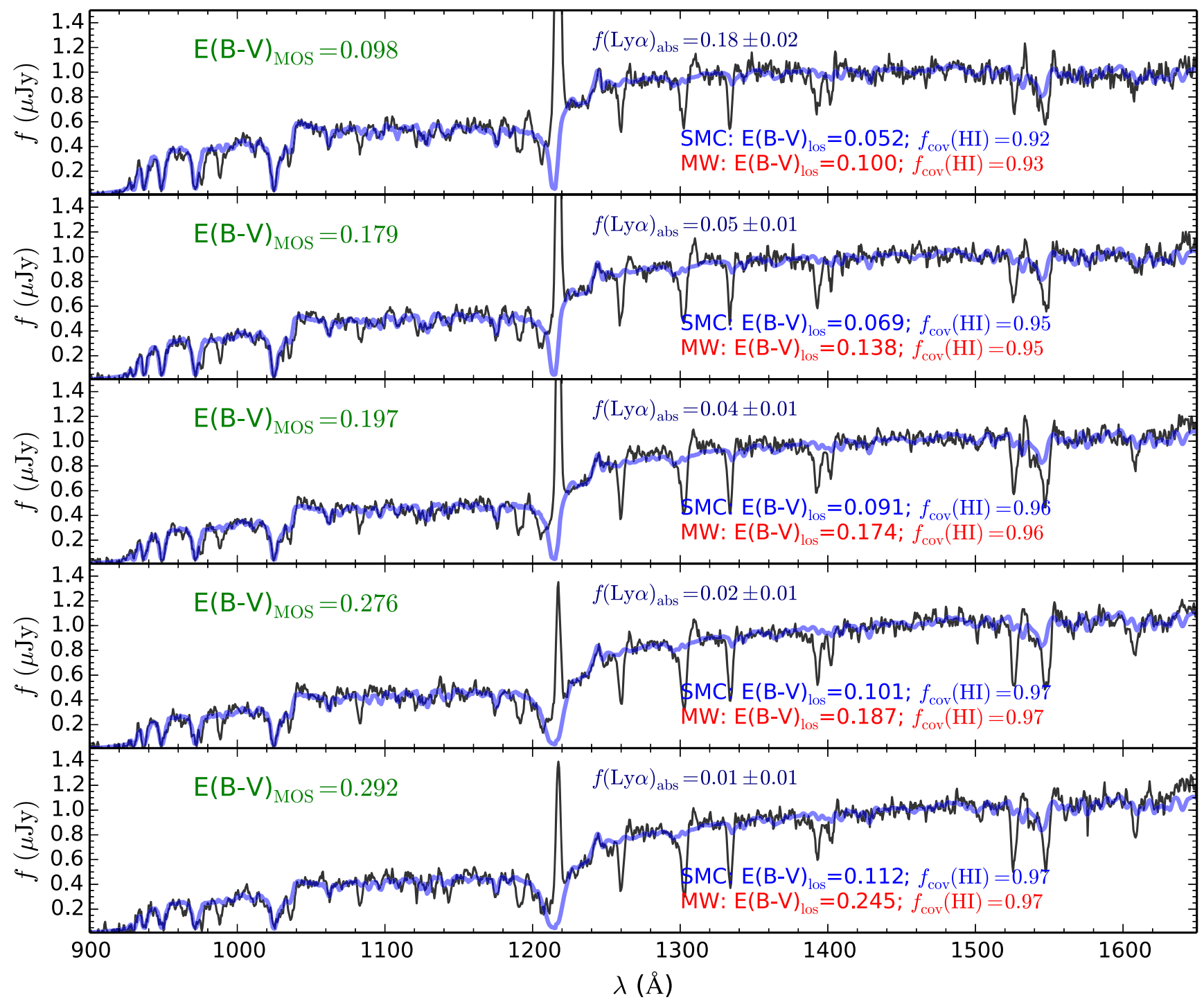

Figure 5. Best-fitting models assuming a non-unity covering fraction of neutral gas and dust, and the SMC extinction curve (blue), to the composite spectra (black), in five bins of $E(B-V)$. The fits assuming the MW extinction curve have been omitted for clarity, but they are similar to those obtained with the SMC extinction curve. The best-fitting $E(B-V)$ assuming the updated Reddy et al. (2015) attenuation curve and a unity covering fraction of dust are indicated in the upper left-hand corner of each panel. Also indicated are the best-fit $E(B-V)_{\text {los }}$ and $f_{\text {cov }}(\mathrm{HI})$, assuming the SMC and Milky Way extinction curves. Uncertainties on the parameters are listed in Table 2, and were estimated by perturbing the composite spectra according to their error spectra and re-fitting the models (see text). Each panel indicates the corresponding absolute escape fraction of Ly $\alpha$ flux.

Table 2

Best-fit $E(B-V)$ and $f_{\text {cov }}(\mathrm{H} \mathrm{I})$

\begin{tabular}{lccccccc}
\hline \hline$E(B-V)_{\mathrm{MOS}^{\mathrm{a}}}$ & $E(B-V)_{\text {Calz }^{\mathrm{a}}}$ & $E(B-V)_{\operatorname{los} / \mathrm{SMC}}{ }^{\mathrm{b}}$ & $f_{\text {cov }}(\mathrm{H} \mathrm{I})^{\mathrm{b}}$ & $\log \left[N(\mathrm{H} \mathrm{I}) / \mathrm{cm}^{-2}\right]^{\mathrm{b}}$ & $E(B-V)_{\operatorname{los} / \mathrm{Mw}}{ }^{\mathrm{c}}$ & $f_{\operatorname{cov}(\mathrm{H} \mathrm{I})^{\mathrm{c}}} \log \left[N(\mathrm{H} \mathrm{I}) / \mathrm{cm}^{-2}\right]^{\mathrm{c}}$ \\
\hline $0.098 \pm 0.005$ & $0.104 \pm 0.006$ & $0.052 \pm 0.002$ & 0.92 & 20.3 & $0.100 \pm 0.002$ & 0.93 \\
$0.179 \pm 0.005$ & $0.184 \pm 0.006$ & $0.069 \pm 0.002$ & 0.95 & 20.3 & $0.138 \pm 0.002$ & 0.95 \\
$0.197 \pm 0.005$ & $0.203 \pm 0.008$ & $0.091 \pm 0.001$ & 0.96 & 20.5 & $0.174 \pm 0.002$ & 0.96 \\
$0.276 \pm 0.006$ & $0.285 \pm 0.005$ & $0.101 \pm 0.001$ & 0.97 & 21.0 & $0.187 \pm 0.004$ & 0.97 & 20.5 \\
$0.292 \pm 0.006$ & $0.301 \pm 0.007$ & $0.112 \pm 0.001$ & 0.97 & 21.0 & $0.245 \pm 0.004$ & 0.97 & 21.2 \\
\hline
\end{tabular}

Notes.

${ }^{a}$ Continuum reddening assuming the updated Reddy et al. (2015) and Calzetti et al. (2000) attenuation curves.

${ }^{\mathrm{b}}$ Best-fitting $E(B-V)_{\operatorname{los}}, f_{\text {cov }}(\mathrm{H} \mathrm{I})$, and $\log \left[N(\mathrm{H} \mathrm{I}) / \mathrm{cm}^{-2}\right]$, assuming a non-unity covering fraction of neutral gas and dust and the SMC extinction curve. The uncertainties in the $\mathrm{H}$ I covering fractions are $\sigma\left(f_{\text {cov }}(\mathrm{H} \mathrm{I})\right)=0.01$. The uncertainties in $\log \left[N(\mathrm{H} \mathrm{I}) / \mathrm{cm}^{-2}\right] \operatorname{are} \sigma\left(\log \left[N(\mathrm{H} \mathrm{I}) / \mathrm{cm}^{-2}\right]\right)=0.2 \mathrm{dex}$.

${ }^{\mathrm{c}}$ Best-fitting $E(B-V)_{\mathrm{los}}, f_{\mathrm{cov}}(\mathrm{H} \mathrm{I})$, and $\log \left[N(\mathrm{H} \mathrm{I}) / \mathrm{cm}^{-2}\right]$, assuming a non-unity covering fraction of neutral gas and dust and the Milky Way extinction curve. The uncertainties in the $\mathrm{H}$ I covering fractions are $\sigma\left(f_{\text {cov }}(\mathrm{H} \mathrm{I})\right)=0.01$. The uncertainties in $\log \left[N(\mathrm{H} \mathrm{I}) / \mathrm{cm}^{-2}\right] \operatorname{are} \sigma\left(\log \left[N(\mathrm{H} \mathrm{I}) / \mathrm{cm}^{-2}\right]\right)=0.2 \mathrm{dex}$. 

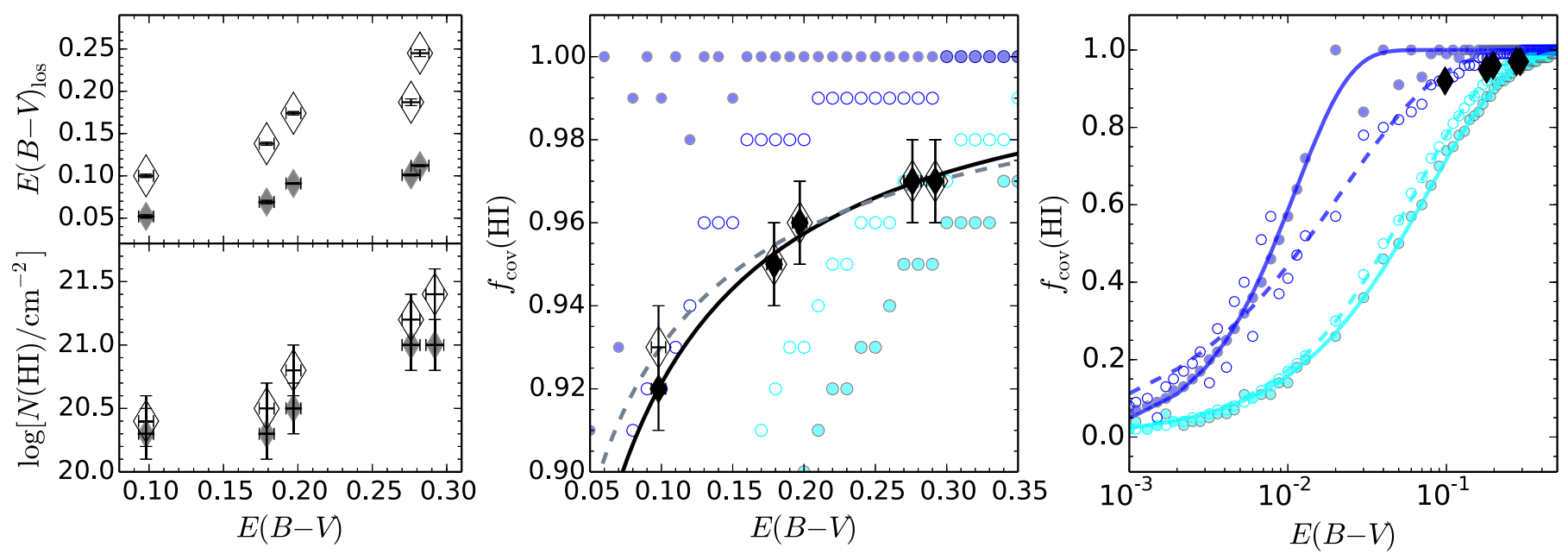

Figure 6. Left: relation between continuum and line-of-sight reddening (top), and continuum reddening and column density of neutral hydrogen (bottom), assuming a non-unity covering fraction of dust, and the SMC ( filled diamonds) and Milky Way (open diamonds) extinction curves. Middle: relation between H I covering fraction and $E(B-V)$. The filled and open diamonds show the measurements obtained directly from the composite spectra assuming the SMC and Milky Way extinction curves, respectively. The solid and dashed lines indicate the best fits to these measurements. The circles denote model expectations of the relationship between the covering fraction and $E(B-V)$. The cyan and blue circles denote the results when assuming the updated and original Reddy et al. (2015) attenuation curves, respectively, for computing the continuum reddening. The open and filled circles correspond to the results when we assume the SMC and MW extinction curves, respectively, for computing the covering fractions. Right: same as the middle panel, but showing a greater dynamic range in both continuum reddening and covering fraction. Also indicated are the best-fit relations (solid and dashed lines) to the model points, assuming the functional form expressed in Equation (15).

Table 3

Summary of Correlation Analysis and Fits

\begin{tabular}{llccc}
\hline \hline$x$ variable & $y$ variable & $a$ & $b$ & $\rho^{\mathrm{b}}$ \\
\hline$E(B-V)$ & $f_{\operatorname{cov}}(\mathrm{H} \mathrm{I})$ & $-5.214 \pm 0.736^{\mathrm{d}}$ & $0.312 \pm 0.074^{\mathrm{d}}$ & 0.90 \\
$\log E(B-V)$ & $\log \left[N(\mathrm{H} \mathrm{I}) / \mathrm{cm}^{-2}\right]$ & $1.60 \pm 0.53^{\mathrm{e}}$ & $21.76 \pm 0.39^{\mathrm{e}}$ & 0.04 \\
$\log E(B-V)$ & $\log E(B-V)_{\operatorname{los}}$ & $0.614 \pm 0.025^{\mathrm{f}}$ & $-0.632 \pm 0.015^{\mathrm{f}}$ & 0.85 \\
\hline
\end{tabular}

Notes.

${ }^{\text {a }}$ Parameters are presented for the correlations obtained when assuming the updated Reddy et al. (2015) stellar attenuation curve and the SMC extinction curve. Other combinations of the attenuation/extinction curves result in correlations with similar fits, with similar Pearson correlation coefficients and similar probabilities for null correlations between the considered quantities.

${ }^{\mathrm{b}}$ Pearson correlation coefficient.

${ }^{\mathrm{c}}$ Probability of a null correlation.

${ }^{\mathrm{d}}$ Free parameters $a$ and $b$, as in Equation (15).

e Slope and intercept, denoted by $a$ and $b$, respectively, in the linear fit to $\log \left[N(\mathrm{H} \mathrm{I}) / \mathrm{cm}^{-2}\right]$ versus $\log E(B-V)$.

${ }^{\mathrm{f}}$ Slope and intercept, denoted by $a$ and $b$, respectively, in the linear fit to $\log E(B-V)_{\operatorname{los}}$ versus $\log E(B-V)$.

estimates, and much of the line-of-sight reddening can be attributed to dust in the outflowing ionized gas in highredshift galaxies (see Section 6.2).

\section{INFERENCES FROM Ly $\alpha$ AND THE LOW- AND HIGH- IONIZATION INTERSTELLAR ABSORPTION LINES}

The discussion in the previous section focused on the parameters obtained from fitting the composite spectra, including the continuum and line-of-sight reddening, and the covering fraction and column density of $\mathrm{H} \mathrm{I}$. We now turn our attention to other features in the stacked spectra that can give further insight into the gas covering fraction and the distribution of metals and dust between the neutral and ionized ISM.

\subsection{Lya Escape Fractions}

Given the correspondence between $\operatorname{Ly} \alpha$ strength and reddening/covering fraction noted in previous studies (e.g., Shapley et al. 2003; Kornei et al. 2010; Jones et al. 2013; Wofford et al. 2013; Rivera-Thorsen et al. 2015; Trainor et al. 2015), we calculated the absolute $\operatorname{Ly} \alpha$ escape fraction,

$$
f(\operatorname{Ly} \alpha)_{\mathrm{abs}}=\frac{L(\operatorname{Ly} \alpha)_{\mathrm{obs}}}{L(\operatorname{Ly} \alpha)_{\mathrm{int}}}
$$

where $L(\operatorname{Ly} \alpha)_{\text {obs }}$ and $L(\operatorname{Ly} \alpha)_{\text {int }}$ are the average observed and intrinsic $\operatorname{Ly} \alpha$ line luminosities, respectively, for the galaxies contributing to each composite shown in Figure 5. The observed line luminosity for each spectral stack was calculated as follows. We first redshifted the composite to the mean redshift of objects contributing to that composite. The spectrum was then converted to $f_{\lambda}$ units. The observed Ly $\alpha$ line luminosity was calculated by directly integrating the flux over just the Ly $\alpha$ emission profile (i.e., from the points where the emission line intersects with the underlying Ly $\alpha$ absorption profile) using the IRAF task splot. Naturally, this calculation will not account for the potentially large fraction of $\operatorname{Ly} \alpha$ (up to 80\%; Steidel et al. 2011) that may be resonantly scattered into a diffuse halo (see discussion below). 


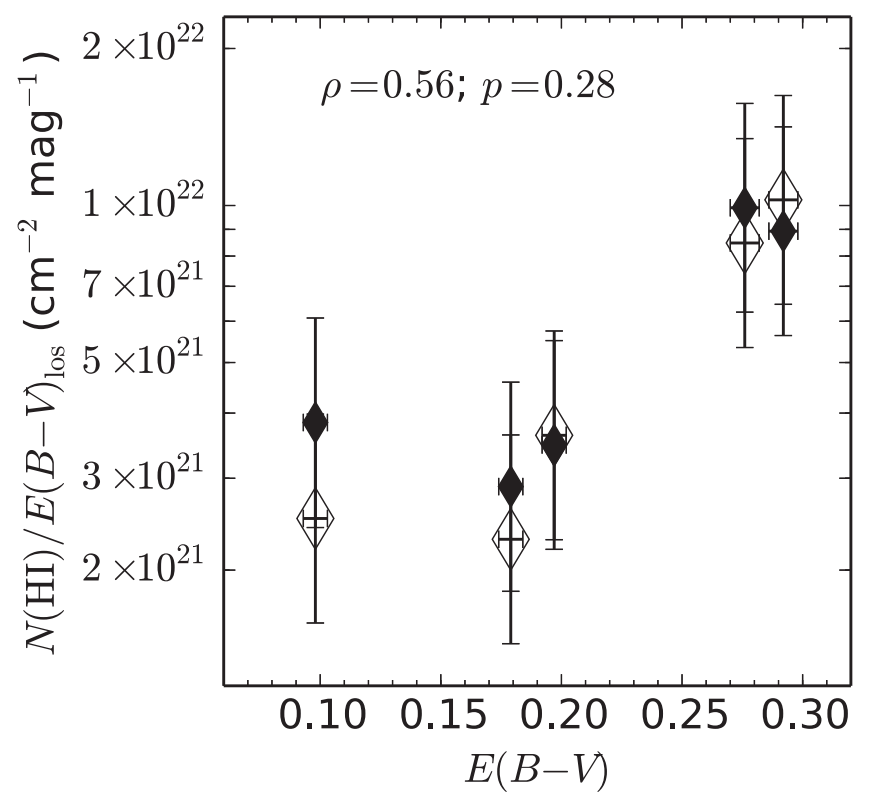

Figure 7. Ratio of the neutral gas column density and line-of-sight reddening, assuming the SMC (filled diamonds) and Milky Way (open diamonds) extinction curves, as a function of continuum reddening. The Pearson correlation coefficient and probability of a null correlation between $N(\mathrm{H} \mathrm{I}) / E(B-V)_{\text {los }}$ and continuum reddening are indicated in the panel for the SMC case, and are similar to those obtained for a Milky Way extinction curve.

The intrinsic Ly $\alpha$ line luminosity was estimated from the SFR. The SFR was calculated using the UV luminosity density at $1500 \AA$, corrected for dust based on the average $E(B-V)$ of objects contributing to each composite and assuming the Reddy et al. (2015) attenuation curve ${ }^{11}$, and converted to SFR assuming the Kennicutt (1998) relation for a Salpeter (1955) IMF. The intrinsic Ly $\alpha$ luminosity was then estimated from the SFR using a modified form of the relation given in Kennicutt (1998), where we assumed that the intrinsic ratio of the Ly $\alpha$-to$\mathrm{H} \alpha$ line intensities is $f(\mathrm{Ly} \alpha) / f(\mathrm{H} \alpha) \simeq 8.7$ for Case $\mathrm{B}$ recombination and $T_{\mathrm{e}}=10^{4} \mathrm{~K}$. This yields the following relationship between SFR and Ly $\alpha$ line luminosity, again assuming a Salpeter (1955) IMF:

$$
L(\operatorname{Ly} \alpha)_{\text {int }}\left[\operatorname{erg~s}^{-1}\right]=\frac{\operatorname{SFR}\left[M_{\odot} \mathrm{yr}^{-1}\right]}{9.1 \times 10^{-43}} .
$$

The measurement error in the mean escape fraction was estimated by perturbing the composite many times according to its error spectrum, and calculating the observed and intrinsic $\operatorname{Ly} \alpha$ luminosities for each of these realizations. Note that the observed Ly $\alpha$ line luminosity and SFR are calculated using the spectrum itself; we made no corrections for slit loss, and thus the escape fractions derived here assume that the Ly $\alpha$ and UV continuum fluxes arise from spatially coincident regions. The resulting $f(\operatorname{Ly} \alpha)_{\mathrm{abs}}$ are indicated for each composite in Figure 5.

Not surprisingly, $f(\operatorname{Ly} \alpha)_{\mathrm{abs}}$ correlates with continuum reddening (and hence with $f_{\text {cov }}(\mathrm{H} \mathrm{I})$; Figure 8; see also Steidel et al. 2010) with a Pearson correlation coefficient of $\rho=-0.97$ and a probability of a null correlation of $p=0.005$. For four of the five $E(B-V)$ bins, the absolute escape fraction of Ly $\alpha$

\footnotetext{
11 These are similar to the dust correction factors obtained with the Calzetti et al. (2000) attenuation curve.
}

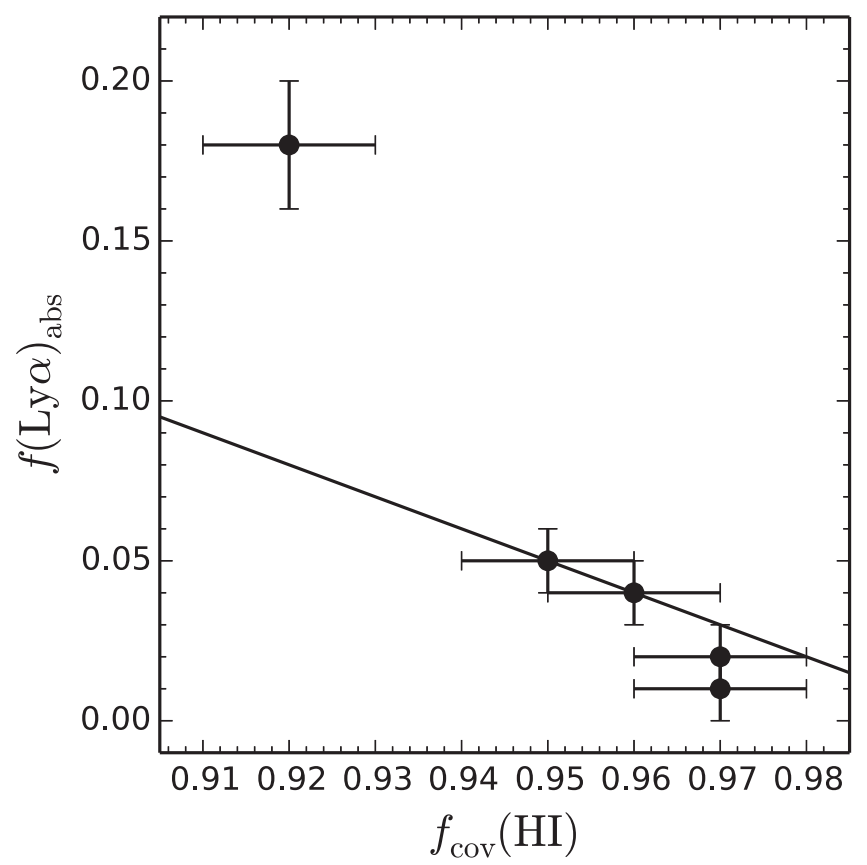

Figure 8. Variation of the absolute escape fraction of Ly $\alpha$ photons $\left(f(\operatorname{Ly} \alpha)_{\mathrm{abs}}\right)$ with neutral gas covering fraction, $f_{\text {cov }}(\mathrm{HI})$. Errors were determined by perturbing the composite spectra many times according to the error spectra and recalculating $f(\mathrm{Ly} \alpha)_{\mathrm{abs}}$ and $f_{\mathrm{cov}}(\mathrm{H} \mathrm{I})$. The solid line shows the relation $f(\mathrm{Ly} \alpha)_{\mathrm{abs}}=1-f_{\text {cov }}(\mathrm{H} \mathrm{I})$, assuming that the Ly $\alpha$ photons escape through clear sightlines in the ISM. Note that the Ly $\alpha$ escape fractions shown here do not include the potentially large fraction of Ly $\alpha$ photons that may be resonantly scattered out of the slit aperture (see text).

photons agrees well with our expectation if such photons only escaped through clear sightlines in the ISM; namely, $f(\mathrm{Ly} \alpha)_{\mathrm{abs}} \approx 1-f_{\mathrm{cov}}(\mathrm{H} \mathrm{I})$ (Figure 8 ). The bluest bin of $E(B-V)$ exhibits a larger $f(\operatorname{Ly} \alpha)$ abs than this expectation. This may suggest that a significant fraction of the Ly $\alpha$ flux may be resonantly scattered into the spectroscopic slit aperture and/ or may arise from Ly $\alpha$ photons scattering out of resonance, though we note that the kinematic profile of the Ly $\alpha$ line for the galaxies in the bluest $E(B-V)$ bin is not substantially different than that of the other bins. Moreover, it is unclear why the fraction of Ly $\alpha$ photons scattered into the spectroscopic slit would be much larger for galaxies with bluer $E(B-V)$. A more detailed assessment of how $\operatorname{Ly} \alpha$ photons escape these galaxies (as judged through the kinematic profile of the line) must await more precise systemic redshifts derived from nebular emission lines.

In the context of Figure 3, one might expect the fraction of Ly $\alpha$ emission exiting through low column density sightlines to correlate more directly with $f_{\text {cov }}(\mathrm{H} \mathrm{I})$ than the diffuse Ly $\alpha$ component; the latter is not accounted for in our calculation. However, based on the results of the previous section, both $N(\mathrm{H} \mathrm{I})$ and $E(B-V)_{\text {los }}$ increase in tandem with $f_{\text {cov }}(\mathrm{H} \mathrm{I})$. Hence, Ly $\alpha$ photons that diffuse far from the sites of star formation face an increasing probability of absorption by dust with increasing $f_{\text {cov }}(\mathrm{HI})$, implying that the fraction of Ly $\alpha$ scattered into a diffuse halo and ultimately exiting the ISM/ CGM of the galaxy is also likely to correlate with $f_{\text {cov }}(\mathrm{H} \mathrm{I})$. This inference could have also been deduced from the results of Steidel et al. (2011), who find that-when considering resonantly scattered $\operatorname{Ly} \alpha$ - the attenuation of $\operatorname{Ly} \alpha$ photons is consistently larger than that of the UV continuum, and the fraction of the total $\operatorname{Ly} \alpha$ produced from star formation that 

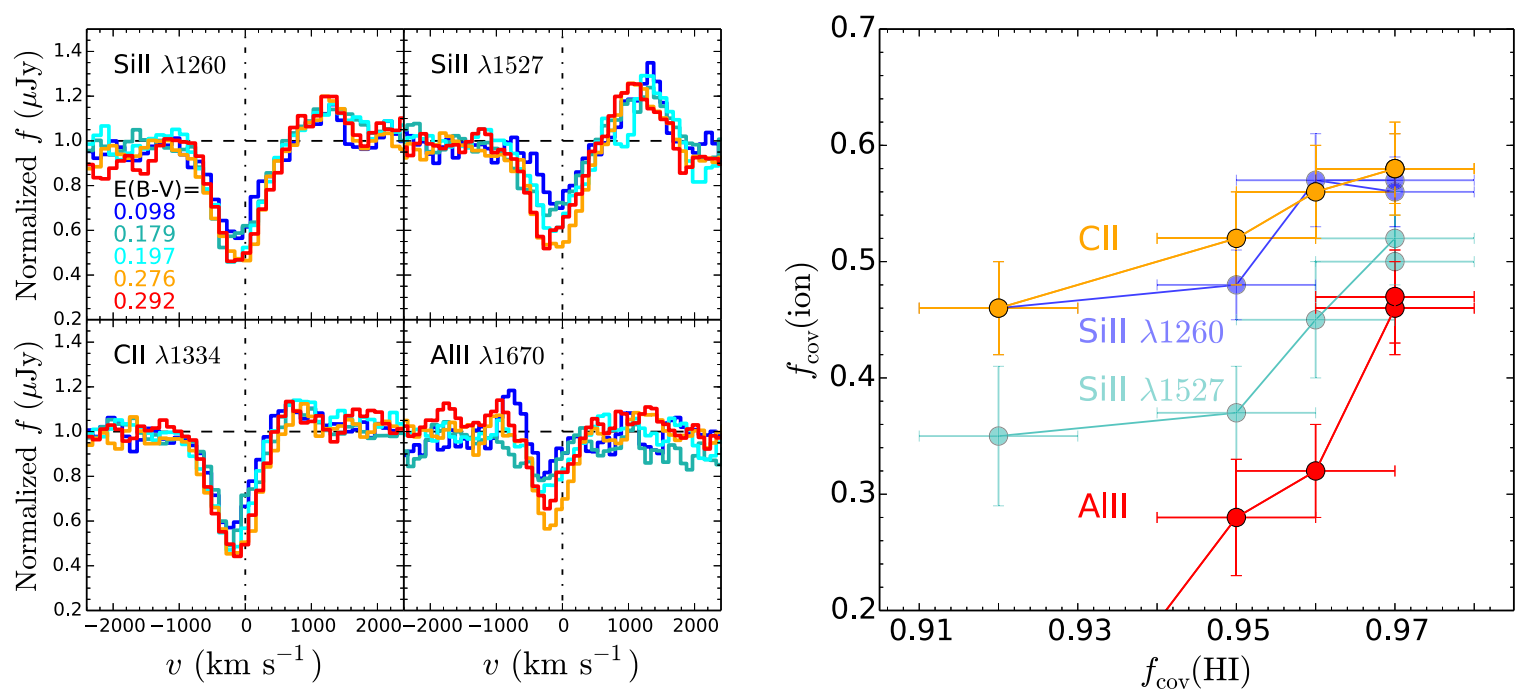

Figure 9. Left: normalized composite spectra in different bins of $E(B-V)$ for several strong low-ionization interstellar absorption lines, including Si II $\lambda 1260$, Si II $\lambda 1527, \mathrm{C}$ II $\lambda 1334$, and Al II $\lambda 1670$. The normalized composites were constructed by dividing the spectra shown in Figure 5 by their best-fitting models. In all cases, the bulk of the absorbing gas is blueshifted by $\approx 300 \mathrm{~km} \mathrm{~s}^{-1}$, similar to that observed for the H I lines. Additionally, the two Si II transitions at 1260 and $1527 \AA$ are saturated and suggest a covering fraction of Si II-enriched material between 35\% and 60\%, and increasing with continuum reddening (see also Shapley et al. 2003). The other low-ionization lines indicate similar covering fractions, assuming that these transitions are also optically thick. Right: the derived covering fractions for Si II $\lambda 1260$ (blue points), Si II $\lambda 1527$ (cyan points), C II $\lambda 1334$ (orange points), and $\mathrm{Al}$ II $\lambda 1670$ (red points), vs. $f_{\text {cov }}$ (H I). The errors in the covering fraction were deduced by perturbing the normalized composite spectra by the normalized error spectra many times, and, each time, locating the point of maximum absorption (minimum flux). The dispersion in the minimum fluxes then determined the errors in the covering fractions for each of the composites and ionic species.

diffuses into a halo correlates inversely with the spectroscopic Ly $\alpha$ flux. At any rate, the computed $f(\operatorname{Ly} \alpha)_{\text {abs }}$ imply that the selection of galaxies based on strong Ly $\alpha$ emission will also tend to isolate those with lower gas covering and higher $\mathrm{LyC}$ escape fractions. In accordance with expectations, the conditions that give rise to strong Ly $\alpha$ emission also facilitate the escape of LyC radiation (e.g., Dijkstra et al. 2016).

\subsection{Interstellar Absorption Lines}

Given the prevalent use of low-ionization interstellar absorption lines as proxies for the neutral gas covering fraction in high-redshift galaxies (e.g., Shapley et al. 2003; Jones et al. 2013; Alexandroff et al. 2015; Trainor et al. 2015), it is instructive to directly compare the covering fractions deduced from the low-ionization lines with those computed from the depth of the H I lines. To accomplish this, the five composites shown in Figure 5 were divided by their respective best-fitting models to produce normalized composites. This method of normalization has the advantage of removing any stellar absorption components (e.g., C II $\lambda 1334$ ) that may affect equivalent width measurements of the interstellar absorption lines. The normalized composites were transformed into velocity space around the Si II $\lambda 1260$, Si II $\lambda 1527$, C II $\lambda 1334$, and $\mathrm{Al}$ II $\lambda 1670$ IS lines. These include the strongest and most commonly used IS lines observed in the rest-frame UV spectra of high-redshift galaxies.

These features are shown in Figure 9, with the different colors indicating the normalized spectrum in each bin of $E(B-V)$. If the two Si II transitions at 1260 and $1527 \AA$ are optically thin, then the ratio of their equivalent widths will be $W_{1260} / W_{1527} \gtrsim 6$. The observed ratio is $W_{1260} / W_{1527} \simeq 1$, implying that the lines are saturated and hence their depths are sensitive to the covering fraction of Si II-enriched material. Other strong low-ionization IS absorption features are shown in Figure 9, including $\mathrm{C}_{\text {II }} \lambda 1334$, and $\mathrm{Al}$ II $\lambda 1670$ lines. The similarity in the velocity widths and depths of these additional lines to those of the saturated Si II transitions discussed above suggests that the former may be also arise from the same gas as the latter. If this is the case, then collectively the depths of the low-ionization lines indicate a covering fraction that rises from $f_{\text {cov }}($ ion $) \approx 0.30$ to 0.65 proceeding from the bluest to reddest $E(B-V)$ bin (see also Shapley et al. 2003). The errors in $f_{\text {cov }}$ (ion) were calculated by perturbing many times the normalized composite spectra by the normalized error spectra, and measuring the depth of the absorption lines for each of these realizations. The dispersion in the absorption line depths yielded the measurement uncertainty in $f_{\text {cov }}$ (ion), but of course does not include systematic errors associated with the specific model for the continuum level.

The covering fractions of the low-ionization species are correlated with, but are systematically lower than, those derived from the H I lines (lower right panel of Figure 9). There are several possible explanations for why the low-ionization lines suggest lower covering fractions. First, if most of the metals inhabit narrow and unresolved absorption systems, then the depth of the metal lines will underpredict the covering fraction. Likewise, if the metal-enriched gas has a substantial velocity gradient across the face of the galaxy, then the derived covering fractions may be lower limits (e.g., see discussion in Section 4.3). While we cannot definitively rule out either of these possibilities, the similarity in the blueshift $\left(\approx 200 \mathrm{~km} \mathrm{~s}^{-1}\right)$ and velocity spread (FWHM $\approx 800 \mathrm{~km} \mathrm{~s}^{-1}$; e.g., compare Figure 9 to the right panel of Figure 4) of the low-ionization and $\mathrm{HI}$ lines suggests that the two components are kinematically coupled, where the former covers a smaller portion of the galaxy's continuum. Such a configuration may arise if dense and metal-enriched pockets of gas are embedded within the outflowing H I. Consequently, some substantial fraction of the outflowing gas may be metal-poor.

Additionally, we note that $f_{\text {cov }}$ (ion) increases by a factor of $\approx 1.5$ in response to a small $(\approx 5 \%)$ increase in $f_{\text {cov }}(\mathrm{H} \mathrm{I})$. This behavior suggests that as $f_{\mathrm{cov}}(\mathrm{H} \mathrm{I})$ increases, a larger fraction 

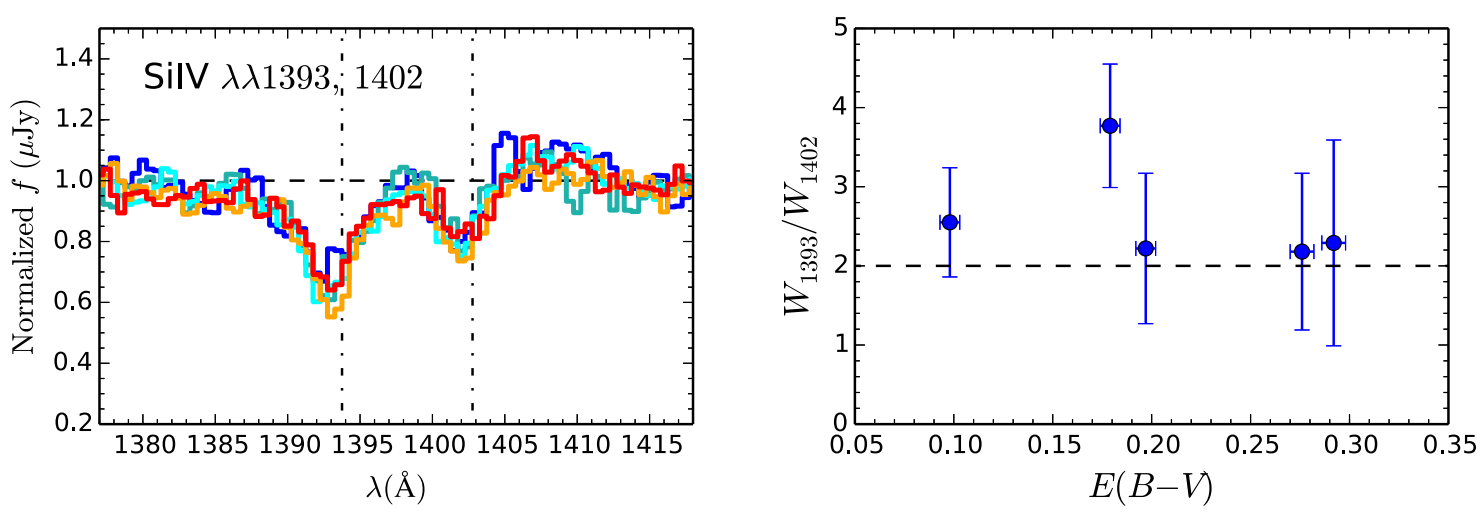

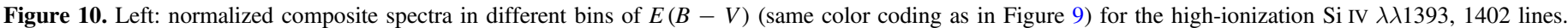
Right: the ratios of the equivalent widths of the two lines as a function of $E(B-V)$ for the five composites suggest an optical depth $\tau($ Si IV) $\simeq 1$.

of the neutral gas is enriched with Si II, implying a corresponding increase in the average dust-to-gas ratio of the outflowing gas. Notwithstanding the fact that the line-of-sight reddening will be sensitive to the total column of dust (including that of the ionized gas), an increase in the dust-togas ratio of the outflowing gas for objects with redder continuum would result in a strong correlation between $E(B-V)_{\text {los }}$ and $E(B-V)$, consistent with what we actually observe (left panel of Figure 6).

Figure 10 shows the profiles of the two Si IV $\lambda \lambda 1393,1402$ transitions for each bin of reddening. As the ionization potential for $\mathrm{SiIV}(q=33.5 \mathrm{eV})$ is much higher than that required for $\mathrm{H}$-ionization, the associated transitions arise from $\mathrm{H}$-ionized gas. The lines are blueshifted by $\approx 160 \mathrm{~km} \mathrm{~s}^{-1}$, slightly smaller than the blueshifts derived from the lowionization and $\mathrm{H}$ I lines, though a more detailed comparison of the kinematics of the neutral, low-, and high-ionization gas will rely on future precise measurements of the systemic redshifts of galaxies in our sample. The ratio of the equivalent widths of the Si IV lines is $W_{1393} / W_{1402} \approx 2$, indicating that the absorption is consistent within the errors of being on the linear part of the curve of growth. As the depths of the lines do not correlate obviously with the reddening of the continuum, we surmise that the outflowing ionized gas has an optical depth $\tau \simeq 1$. Note that the errors in $W_{1393} / W_{1402}$ do not rule the possibility of the lines being saturated, in which case the covering fraction of the ionized material is $f_{\text {cov }}(\mathrm{Si}$ IV) $\gtrsim 30 \%$. More generally, the presence of these high-ionization IS absorption lines suggests an ionized medium mixed with metals and dust.

\subsection{Summary of Results from Ly $\alpha$ and Interstellar Absorption Lines}

To summarize, we find that the absolute escape fraction of Ly $\alpha$ photons, $f(\operatorname{Ly} \alpha)_{\text {abs }}$, inversely correlates with $f_{\text {cov }}(\mathrm{H} \mathrm{I})$, suggesting that the neutral gas covering fraction plays a significant role in modulating the escape of Ly $\alpha$ photons. The low-ionization interstellar doublet $\mathrm{Si}$ II $\lambda \lambda 1260,1527$ is saturated, exhibits similar absorption depths and velocity profiles to those of other low-ionization IS absorption lines, and suggests a covering fraction of low-ionization material, $f_{\text {cov }}$ (ion), that increases with continuum reddening (and $\left.f_{\text {cov }}(\mathrm{H} \mathrm{I})\right)$ from $\approx 0.30$ for the bluest galaxies in our sample to $\approx 0.65$ for the reddest ones. However, $f_{\text {cov }}$ (ion) is systematically lower than $f_{\text {cov }}(\mathrm{HI})$, while the low-ionization lines have similar blueshifts and velocity profiles as the $\mathrm{H}$ I lines, suggesting that galaxy outflows consist in part of a neutral medium embedded with clumps of metal-enriched gas. Moreover, the rate of increase of $f_{\text {cov }}$ (ion) relative to $f_{\text {cov }}(\mathrm{H} \mathrm{I})$ implies that the average column of dust increases with covering fraction, consistent with our observation that the line-of-sight reddening correlates with continuum reddening. These results imply that a significant fraction of the neutral medium may be chemically pristine, and that low-ionization interstellar absorption lines may severely underpredict the neutral gas covering fraction. A similar analysis for the high-ionization absorption lines, namely Si IV $\lambda \lambda 1393$, 1402, suggests that the highionization gas is not optically thick and has $f_{\text {cov }}(\mathrm{Si}$ IV $) \gtrsim 30 \%$.

\section{CONNECTION BETWEEN CONTINUUM REDDENING AND ESCAPE OF IONIZING PHOTONS}

\subsection{Gas Covering and LyC Escape Fractions}

LyC photons may be extinguished by dust absorption or through hydrogen photoelectric absorption. In the case of dust absorption, our new measurement of the far-UV shape of the dust curve at high redshift, presented in Paper I, implies a lower attenuation of LyC photons than those inferred from simple extrapolations of the Calzetti et al. (2000) and Reddy et al. (2015) attenuation curves. Alternatively, the high column densities $\left(\log \left[N(\mathrm{H} \mathrm{I}) / \mathrm{cm}^{-2}\right] \gtrsim 20.3\right)$ inferred from the fitting presented in Section 5 suggest that the covered portions of a galaxy are optically thick to LyC radiation, with implied optical depths at $900 \AA$ of $\tau_{900} \simeq 1150$. Thus, for the average galaxy, photoelectric absorption rather than dust dominates the depletion of LyC photons. Similar results have been suggested by simulations of escaping ionizing radiation from high-redshift galaxies (Gnedin et al. 2008; Razoumov \& SommerLarsen 2010; Ma et al. 2016). Accordingly, in the geometry illustrated in Figure 3, LyC photons will only escape from dustfree holes in the ISM. The very high optical depth of the covering neutral gas suggests that Ly $\alpha$ photons will also predominantly escape from regions cleared of dust and gas, an expectation that is verified by the strong correlation between $f(\operatorname{Ly} \alpha)_{\text {abs }}$ and reddening/covering fraction (Section 6.1).

LyC extinction may have a more significant effect in galaxies where the radiation field is sufficient to completely ionize the hydrogen (i.e., corresponding to a "density-bounded" case; see Zackrisson et al. 2013), but such galaxies must be relatively rare in our sample given the apparent frequency of 
high column density neutral gas as reflected in the composite spectra. We can connect the gas covering fraction to the absolute escape fraction of $\mathrm{LyC}$ photons, where the latter is commonly written as:

$$
\begin{aligned}
f(\mathrm{LyC})_{\mathrm{abs}}= & \exp \left[-\tau_{\mathrm{ISM}}(\mathrm{LyC})\right] \\
& \times 10^{-0.4 k(\mathrm{LyC}) E(B-V)} .
\end{aligned}
$$

The first term in this expression accounts for the opacity of the ISM, where $\tau_{\text {ISM }}(\mathrm{LyC})$ is the optical depth at LyC wavelengths. The second term accounts for the obscuration of $\mathrm{LyC}$ photons by dust, where $E(B-V)$ is the reddening and $k(\mathrm{LyC})$ is the dust attenuation curve evaluated at $\mathrm{LyC}$ wavelengths. In the case where the dust and neutral gas completely cover the continuum, the opacity of the ISM can be combined with the dust attenuation curves calculated in Paper I to compute $f(\mathrm{LyC})_{\mathrm{abs}}$.

In the case of incomplete covering, the absolute escape fraction of $\mathrm{LyC}$ photons can be written as:

$$
\begin{aligned}
f(\mathrm{LyC})_{\mathrm{abs}}= & f_{\mathrm{cov}}(\mathrm{H} \mathrm{I}) \exp \left[-\tau_{\mathrm{ISM}}^{\mathrm{cov}}(\mathrm{LyC})\right] \\
& \times 10^{-0.4 k(\mathrm{LyC}) E(B-V)_{\mathrm{los}}^{\mathrm{cov}}} \\
& +\left(1-f_{\mathrm{cov}}(\mathrm{H} \mathrm{I} \mathrm{I}) \exp \left[-\tau_{\mathrm{ISM}}^{\mathrm{uncov}}(\mathrm{LyC})\right]\right. \\
& \times 10^{-0.4 k(\mathrm{LyC}) E(B-V)_{\mathrm{los}}^{\text {uncov }}},
\end{aligned}
$$

where the first and second terms correspond to the covered and uncovered portions contributing to the galaxy spectrum, respectively. In this expression, $k(\mathrm{LyC})$ is a line-of-sight extinction curve evaluated at LyC wavelengths, and $E(B-V)_{\mathrm{los}}^{\mathrm{cov}}$ and $E(B-V)_{\mathrm{los}}^{\mathrm{uncov}}$ indicate the line-of-sight reddening appropriate to the covered and uncovered continuum, respectively.

Similarly, the absolute escape fraction of UV continuum photons (e.g., typically measured between 1500 and $1700 \AA$ ) is

$$
f(\mathrm{UV})_{\mathrm{abs}}=10^{-0.4 k(\mathrm{UV}) E(B-V)},
$$

where $k(\mathrm{UV})$ refers to the attenuation curve relevant for the stellar continuum, assuming a unity covering fraction of dust. ${ }^{12}$

These equations are cast most conveniently in terms of the "relative" escape fraction defined as

$$
f_{\text {rel }}=\frac{f(\mathrm{LyC})_{\mathrm{abs}}}{f(\mathrm{UV})_{\mathrm{abs}}}
$$

(Steidel et al. 2001). The relationship between the relative escape fraction and the observed ratio of LyC-to-UV flux

\footnotetext{
${ }^{12}$ Note that we can also write $f(\mathrm{UV})_{\text {abs }}$ as two terms that refer to the covered and uncovered portions of the continuum, analogous to Equation (6):

$$
\begin{aligned}
& f(\mathrm{UV})_{\mathrm{abs}}=f_{\mathrm{cov}}(\mathrm{H} \mathrm{I}) \times 10^{-0.4 k(\mathrm{UV}) E(B-V)_{\mathrm{los}}^{\operatorname{cov}}} \\
& +\left(1-f_{\mathrm{cov}}(\mathrm{H} \mathrm{I})\right) \times 10^{-0.4 k(\mathrm{UV}) E(B-V)_{\mathrm{los}}^{\text {uncov }},}
\end{aligned}
$$

which, in the case of low dust column density for the uncovered portion, can be written as

$$
f(\mathrm{UV})_{\text {abs }} \approx f_{\text {cov }}(\mathrm{H} \mathrm{I}) \times 10^{-0.4 k(\mathrm{UV}) E(B-V)^{\mathrm{cov}}}
$$$$
+\left(1-f_{\text {cov }}(\mathrm{H} \mathrm{I})\right)
$$

where $k(\mathrm{UV})$ now refers to a line-of-sight extinction curve. As the color excess is measured most conveniently assuming a unity covering fraction of dust with a stellar attenuation curve, these equations are of less practical use than Equation (7).
}

density is

$$
\begin{aligned}
f_{\text {rel }}= & \frac{S(\mathrm{LyC})_{\mathrm{obs}} / S(\mathrm{LyC})_{\mathrm{int}}}{S(\mathrm{UV})_{\mathrm{obs}} / S(\mathrm{UV})_{\mathrm{int}}} \\
& \times \exp \left[\tau_{\mathrm{IGM}}(\mathrm{LyC})\right] \times \exp \left[\tau_{\mathrm{CGM}}(\mathrm{CGM})\right],
\end{aligned}
$$

where $S(\mathrm{LyC})_{\mathrm{obs}}$ and $S(\mathrm{UV})_{\mathrm{obs}}$ are the observed flux densities of LyC and UV continuum photons, respectively; $S(\mathrm{LyC})_{\text {int }}$ and $S(\mathrm{UV})_{\text {int }}$ are the intrinsic flux densities of LyC and UV continuum photons, respectively, as typically estimated from stellar population synthesis models; and the last two terms indicate the further depletion of $\mathrm{LyC}$ photons due to the opacity of the IGM and CGM.

Combining Equations (7), (10), and (11) yields the following expression for the observed LyC-to-UV flux density ratio:

$$
\begin{aligned}
{\left[\frac{S(\mathrm{LyC})}{S(\mathrm{UV})}\right]_{\mathrm{obs}}=} & {\left[\frac{S(\mathrm{LyC})}{S(\mathrm{UV})}\right]_{\mathrm{int}} } \\
& \times \exp \left[-\tau_{\mathrm{IGM}}(\mathrm{LyC})\right] \\
& \times \exp \left[-\tau_{\mathrm{CGM}}(\mathrm{LyC})\right] \\
& \times\left[\frac{f(\mathrm{LyC})_{\mathrm{abs}}}{10^{-0.4 k(\mathrm{UV}) E(B-V)}}\right],
\end{aligned}
$$

where $f(\mathrm{LyC})_{\text {abs }}$ is given by Equation (6).

For the average $z \sim 3$ galaxy in our sample, the first term of Equation (6) is negligible owing to the large neutral gas column density and hence the large value of $\tau_{\mathrm{ISM}}^{\mathrm{cov}}$, as noted above. Hence, if the uncovered portions of the galaxy continuum are characterized by very low gas and dust column densities, as would be expected in the simple geometry of Figure 3, then the absolute escape fraction of LyC photons is simply

$$
f(\mathrm{LyC})_{\mathrm{abs}} \approx 1-f_{\mathrm{cov}}(\mathrm{H} \mathrm{I}) .
$$

We noted in Section 4.3 that $f(\mathrm{LyC})_{\text {abs }}$ may be lower than that indicated by Equation (13). We discuss this further in Section 7.4.3. In the present context, Equation (12) becomes

$$
\begin{aligned}
{\left[\frac{S(\mathrm{LyC})}{S(\mathrm{UV})}\right]_{\mathrm{obs}}=} & {\left[\frac{S(\mathrm{LyC})}{S(\mathrm{UV})}\right]_{\mathrm{int}} } \\
& \times \exp \left[-\tau_{\mathrm{IGM}}(\mathrm{LyC})\right] \\
& \times \exp \left[-\tau_{\mathrm{CGM}}(\mathrm{LyC})\right] \\
& \times\left[\frac{1-f_{\mathrm{cov}}(\mathrm{H} \mathrm{I})}{\left.10^{-0.4 k(\mathrm{UV}) E(B-V)}\right]} .\right.
\end{aligned}
$$

In practice, $f_{\mathrm{cov}}(\mathrm{H} \mathrm{I})$ is difficult to constrain in the absence of direct spectroscopy of the $\mathrm{H} \mathrm{I}$ absorption lines-e.g., as noted in Section 6.2, the neutral gas covering fractions deduced from low-ionization IS absorption lines may severely underpredict those derived from the $\mathrm{HI}$ lines. Thus, the utility of an empirical relation between $f_{\text {cov }}(\mathrm{H} \mathrm{I})$ and $E(B-V)$ becomes obvious: the continuum reddening is relatively easy to measure, even with just a few broadband photometric filters covering the rest-frame UV. As discussed in Section 5.4 and shown in Figure 6 and Table 3, we find a significant correlation between $f_{\text {cov }}(\mathrm{H} \mathrm{I})$ and $E(B-V)$. Thus, a suitable functional relationship between these two variables can be used to cast Equations (6) and (14) in terms of $E(B-V)$. The functional form relating $f_{\mathrm{cov}}(\mathrm{H} \mathrm{I})$ and $E(B-V)$ is the subject of the next subsection. 


\subsection{Functional Form for the Gas Covering Fraction in Terms of Continuum Reddening}

The middle and right panels of Figure 6 show the relationship between $f_{\text {cov }}(\mathrm{HI})$ and $E(B-V)$. The Pearson correlation coefficients when we assume the SMC and Milky Way extinction curves in computing $f_{\text {cov }}(\mathrm{H} \mathrm{I})$ are $\rho=0.90$ and 0.87 , respectively, with probabilities for no correlation between $f_{\text {cov }}(\mathrm{H} \mathrm{I})$ and $E(B-V)$ of $p=0.04$ and 0.05 , respectively. To uncover the functional form of $f_{\text {cov }}(\mathrm{H} \mathrm{I})$ versus $E(B-V)$, we reddened the intrinsic template assuming different values of $E(B-V)$ and the updated and original forms of the Reddy et al. (2015) and Calzetti et al. (2000) attenuation curves. For each of these reddened templates, we determined which combination of the covering fraction and line-of-sight reddening-assuming either the SMC or Milky Way extinction curves-provided the best match in the "Composite $E(B-V)$ " wavelength windows listed in Table 1 , and one additional window covering the LyC region: $\lambda=800-900 \AA$. The model expectations for various assumptions of the reddening and line-of-sight dust curves are shown in the middle and right panels of Figure 6. For clarity, not all of the combinations of attenuation/extinction curves are shown, as they all result in a similar functional behavior between $f_{\text {cov }}(\mathrm{H} \mathrm{I})$ and $E(B-V)$.

As expected, the covering fraction asymptotes to zero and unity for small and large values of the continuum reddening, respectively. Motivated by this behavior, we adopted the following functional form for $f_{\text {cov }}(\mathrm{H} \mathrm{I})$ versus $E(B-V)$ :

$$
f_{\mathrm{cov}}(\mathrm{H} \mathrm{I})=1-\exp \left[a \times E(B-V)^{b}\right],
$$

where $a$ and $b$ are free parameters. Table 3 summarizes the free parameters obtained by fitting the measured values of $f_{\text {cov }}$ and $E(B-V)$ for the updated Reddy et al. (2015) attenuation curve and the SMC extinction curve applicable to the continuum and line-of-sight reddening, respectively.

To compute these fits, we randomly perturbed the color excess and covering fraction measurements according to their respective errors and fit each of these realizations with Equation (15). The average and standard deviation in the best-fit parameters among these realizations are the values reported in Table 3. The $68 \%$ confidence intervals in the fits were computed as the $1 \sigma$ dispersion in the fits to the realizations at each value of $E(B-V)$. These confidence intervals do not vary significantly over the range of $E(B-V)$ probed in our study for different assumptions of the attenuation curve, as the fits are forced to go through the measured data.

Finally, we can combine Equations (14) and (15) to obtain an expression for the observed LyC-to-UV flux density ratio in terms of $E(B-V)$ for the geometry illustrated in Figure 3:

$$
\begin{aligned}
\left\langle\frac{S(\mathrm{LyC})}{S(\mathrm{UV})}\right\rangle_{\mathrm{obs}}= & \left\langle\frac{S(\mathrm{LyC})}{S(\mathrm{UV})}\right\rangle_{\mathrm{int}} \\
& \times\left\langle\exp \left[-\tau_{\mathrm{IGM}}(\mathrm{LyC})\right]\right. \\
& \left.\times \exp \left[-\tau_{\mathrm{CGM}}(\mathrm{LyC})\right]\right\rangle \\
& \times\left[\frac{\exp \left[a \times\langle E(B-V)\rangle^{b}\right]}{\left.10^{-0.4 k(\mathrm{UV})\langle E(B-V)\rangle}\right] .}\right.
\end{aligned}
$$

We have written this equation in terms of averages to emphasize that the relation applies for an ensemble of galaxies. Adopting values for the IGM and CGM opacities appropriate for the redshifts of interest allows us to use Equations (6), (12), and (16) in several useful ways, as we discuss in Section 7.4.3.

\subsection{Extending the Model of LyC Escape to Bluer Galaxies}

To uncover the extent to which the relationship between $[S(\mathrm{LyC}) / S(\mathrm{UV})]_{\mathrm{obs}}$ and $E(B-V)$ may change over a larger range of $E(B-V)$ than accessible in the current sample, we examined how several of the other factors relevant in the escape of LyC photons, namely $N(\mathrm{H} \mathrm{I})$ and $E(B-V)_{\text {los }}$, vary with $E(B-V)$. As noted in Section 5.4, and shown in Figure 6 and Table 3, several significant correlations are found between these factors and $E(B-V)$. We fit $\log N(\mathrm{H} \mathrm{I})$ versus $\log E(B-V)$ and $\log E(B-V)_{\operatorname{los}}$ versus $\log E(B-V)$ with linear functions. The best-fitting intercepts and slopes, and their uncertainties, are summarized in Table 3 . We used these linear fits to estimate $N(\mathrm{H} \mathrm{I})$ and $E(B-V)_{\mathrm{los}}$ for galaxies with $E(B-V) \lesssim 0.09$, and used Equation (6) to calculate $f(\mathrm{LyC})_{\mathrm{abs}}$, and Equation (12) to calculate $[S(\mathrm{LyC}) / S(\mathrm{UV})]_{\mathrm{obs}}$. In the next section, we examine how $[S(\mathrm{LyC}) / S(\mathrm{UV})]_{\text {obs }}$ varies with continuum reddening.

\subsection{Practical Applications of the Model of LyC Escape}

\subsubsection{Calculating the Models}

To calculate the predicted $\langle S(\mathrm{LyC}) / S(\mathrm{UV})\rangle_{\text {obs }}$ from our model, we first calculated the expected opacities of the IGM and CGM by considering the neutral gas column density distribution in the $\operatorname{Ly} \alpha$ forest at $z \sim 2.0-2.8$, as well as the excess $\mathrm{HI}$ absorption around $\sim L^{*}$ galaxies at the same redshifts, as quantified in Rudie et al. (2013). Using 1000 random realizations of these column density distributions, we calculate the attenuation of $900 \AA$ photons to be $\left\langle\exp \left[-\tau_{\mathrm{IGM}}(900)\right] \times \exp \left[-\tau_{\mathrm{CGM}}(900)\right]\right\rangle=0.37$ for galaxies at $z=3.05$, corresponding to the mean redshift of the 121 galaxies that contribute to the composite at $\lambda<1150 \AA$. The dispersion in the transmissivity among the 1000 realizations at this wavelength is $\approx 0.33$, implying that the error in the mean transmissivity for the 121 galaxies is $\approx 8.1 \%$.

Next, we use the observed relations listed in Table 3 to estimate $f_{\text {cov }}(\mathrm{H} \mathrm{I}), E(B-V)_{\text {los }}$ and $N(\mathrm{H} \mathrm{I})$, for different values of $E(B-V)$. Thus, Equations (6), (12) and (16) can be written purely in terms of $E(B-V)$, the line-of-sight extinction and attenuation curves evaluated at the wavelengths of LyC and UV photons, respectively, and the intrinsic LyC-toUV flux density ratio. To examine the possible range of models given the constraints imposed by our data, we calculated an "extrapolated" model which assumes that the neutral gas is optically thick to ionizing photons at all values of $E(B-V)$, and that the uncovered portions of the galaxies are effectively free of dust and gas. In this case, the model is given simply by Equation (16). In the "fiducial" model, we assumed that the neutral gas column density varies with $E(B-V)$ according to the relations listed in Table 3. Finally, in the "fixed" model, we assumed that the ratio $N(\mathrm{H} \mathrm{I}) / E(B-V)_{\text {los }}$ is fixed, and that $E(B-V)_{\text {los }}$ scales with $E(B-V)$ according to the relations listed in Table 3. All three models were computed assuming the combination of the updated Reddy et al. (2015) attenuation curve and either the SMC or Milky Way extinction curves, and are shown in Figure 11. Other combinations of the stellar attenuation and line-of-sight extinction curves yield quantitatively similar results (see discussion below). All models shown are computed by taking the UV luminosity density at $1500 \AA$ 


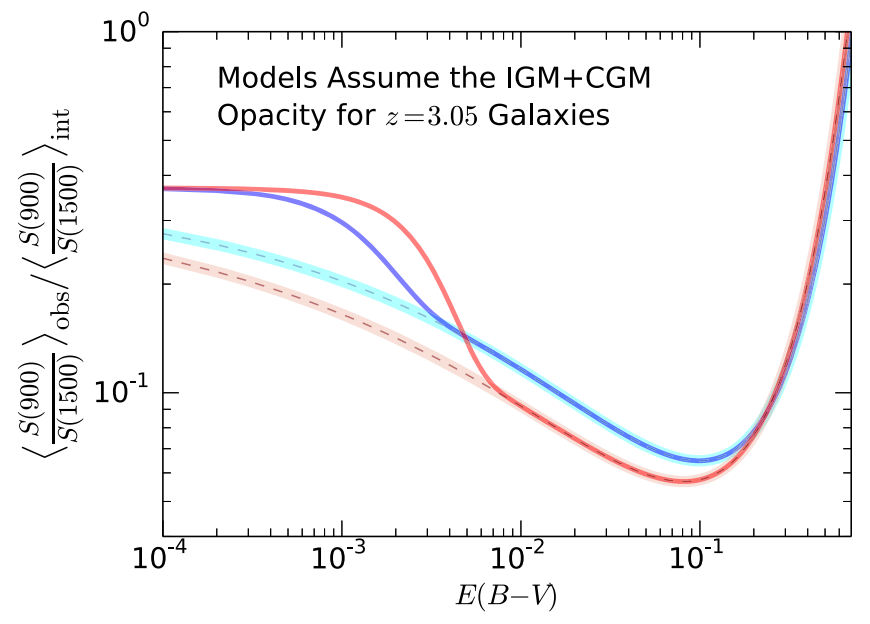

Figure 11. Dependence of the observed 900-to-1500 $\AA$ flux density ratio (normalized by the intrinsic ratio) on continuum color excess, $E(B-V)$, assuming the SMC (blue and cyan lines) or the Milky Way extinction curve (red and light red lines). The solid lines indicate the "fiducial" models where $N(\mathrm{H} \mathrm{I})$ varies with $E(B-V)$ according to the relations listed in Table 3 . The thick shaded curves denote the "extrapolated" models which assume that the covering $\mathrm{H} \mathrm{I}$ is optically thick to ionizing photons for all values of $E(B-V)$. The dashed curves represent the "fixed" models, where $N(\mathrm{H} \mathrm{I}) / E(B-V)_{\operatorname{los}}$ is fixed and $E(B-V)_{\text {los }}$ varies with $E(B-V)$ according to the relation listed in Table 3. All curves assume the combined IGM and CGM opacities appropriate for the average line of sight toward $z \sim 3$ galaxies.

and are cast in terms of the ratio of the observed and intrinsic 900-to-1500 Å flux density ratios.

\subsubsection{Behavior of the Models}

In all of the cases considered here, the extrapolated and fixed models are indistinguishable because a fixed $N(\mathrm{H} \mathrm{I}) /$ $E(B-V)_{\text {los }}$ implies optically thick $\mathrm{H}$ I over the entire range of $E(B-V)=10^{-4}$ to $10^{\circ}$, as shown in Figure 11 . The fiducial model deviates from the other two at $E(B-V) \lesssim 0.01$ where the neutral gas becomes optically thin $\left(N(\mathrm{H} \mathrm{I}) \lesssim 5 \times 10^{17} \mathrm{~cm}^{-2}\right)$, thus allowing the escape of additional LyC photons above the expectations from the extrapolated model (i.e., the covered portion of the galaxies' continua, as illustrated in Figure 3, becomes optically thin). For very blue $E(B-V), f_{\text {cov }}(\mathrm{HI}) \rightarrow 0$ and the observed LyC-to-UV flux density ratio simply converges to the intrinsic value modulated by the combined IGM and CGM opacities. For very red $E(B-V)$, the ratio of the observed and intrinsic LyCto-UV flux density ratios increases to values larger than unity. This can be explained as follows. LyC photons escape the galaxies only through clear sightlines, and the modeling assumes that the dust attenuation of such photons in negligible along these sightlines. Thus, the only way in which the observed LyC flux density depends on the reddening is through the relationship between $f_{\text {cov }}(\mathrm{H} \mathrm{I})$ and $E(B-V)$. The absolute escape fraction of LyC photons, i.e., $1-f_{\text {cov }}(\mathrm{H} \mathrm{I})$, does not decrease as rapidly as the absolute escape fraction of non-ionizing UV photons-as the covering fraction asymptotes to unity for $E(B-V) \rightarrow \infty-$ resulting in a steep rise of the model prediction of $\left[S(900)_{\mathrm{obs}} / S(1500)_{\mathrm{obs}}\right] /\left[S(900)_{\mathrm{int}} / S(1500)_{\mathrm{int}}\right]$

for $E(B-V) \gtrsim 0.3$.

There is negligible variation in the model curve shapes at $E(B-V) \lesssim 0.1$ for different assumptions of the stellar attenuation curve. Thus, in practice, one can simply assume a single model that is invariant to assumptions of the adopted attenuation curve. On the other hand, the offset in the relation between $f_{\text {cov }}(\mathrm{H} \mathrm{I})$ and $E(B-V)$ that arises from different assumptions of the line-of-sight extinction curve (e.g., Figure 6) results in a factor of up to $\approx 1.5$ difference in the predicted $\langle S(900) / S(1500)\rangle_{\mathrm{obs}}$ at a given $E(B-V)$ for $E(B-V) \lesssim 0.10$. This systematic difference may be partly attributed to the lack of data for galaxies bluer than $E(B-V) \simeq 0.1$ with which to constrain the fit between $f_{\text {cov }}(\mathrm{H} \mathrm{I})$ and $E(B-V)$. In the following, we discuss how these model curves may be used to evaluate the possible mechanisms of LyC escape and/or constrain the intrinsic ionizing spectrum of high-redshift galaxies.

\subsubsection{Constraints on the Intrinsic LyC-to-UV Flux Density Ratio}

We preface our discussion by noting that deep far-UV spectra of the kind used for our study would be useful to obtain over a broader dynamic range of $E(B-V)$, in particular focusing on the bluer galaxies that are more analogous to typical galaxies at $z \gtrsim 5$ and which may have larger ionizing escape fractions. FarUV spectra may be used to directly measure the covering fraction of neutral gas at $E(B-V) \lesssim 0.09$ using the technique described in Section 5, and will enable more accurate estimates of the column densities and line-of-sight reddening for such galaxies. Nonetheless, in the following, we illustrate how such a model may be used to discern the mechanisms by which LyC photons escape from high-redshift galaxies.

The most direct application of our model is that it can be used to predict the average relative escape fraction of ionizing photons for a given stellar population model. For example, if we assume $\langle S(900) / S(1500)\rangle_{\text {int }}=1 / 6$, a value typical of that adopted in previous studies (e.g., Siana et al. 2007; Nestor et al. 2011), then a sample of galaxies at $z=3$ with $\langle E(B-V)\rangle=0.15$ would be predicted to have an observed relative escape fraction of $\langle S(900) / S(1500)\rangle_{\mathrm{obs}} \approx 0.011$. The relative escape fraction corrected for attenuation by the IGM and CGM is 0.031 , and the absolute escape fraction is $\approx 5 \%$. We emphasize that the model should only be applied to an ensemble of galaxies where the stellar populations and different configurations of the gas and stars from galaxy-to-galaxy, and the stochasticity in the $\operatorname{Ly} \alpha$ forest along different sightlines, can be averaged out.

A second and potentially powerful application of our model is immediately obvious. Namely, with direct measurements of the average observed LyC-to-UV flux density ratio for an ensemble of galaxies with some average continuum reddening, $\langle E(B-V)\rangle$, one may invert Equation (16) to calculate $\langle S(900) / S(1500)\rangle_{\text {int }}$. The importance of direct empirical constraints on the intrinsic LyC-to-UV flux density ratio is underscored by the fact that the different flavors of stellar population models (e.g., arising from different ages and metallicities for a given star formation history, and/or the treatment of stellar rotation/binarity; Eldridge \& Stanway 2009; Brott et al. 2011; Levesque et al. 2012; Leitherer et al. 2014) predict a factor of $\approx 5$ variation in the intrinsic LyC-to-UV flux density ratios of $\simeq 1.3-6.4$ (e.g., Nestor et al. 2013). The actual range in the predictions of the stellar population models are larger than a factor of $\approx 5$ if we also consider variations in the stellar initial mass function (IMF) and star formation history. Clearly, independent information from the rest-frame UV continuum spectra, rest-frame optical nebular emission line spectra, and/or stellar population modeling may be used to narrow the range of possible $\langle S(\mathrm{LyC}) / S(\mathrm{UV})\rangle_{\text {int }}$ (Steidel et al. 2016). Nevertheless, there is substantial utility in directly 
constraining the intrinsic LyC-to-UV flux density ratio without having to extrapolate the spectra of massive stars to wavelengths blueward of what is typically accessible from observations.

To demonstrate how our model can be used to constrain the intrinsic LyC-to-UV flux density ratio, we considered the recent measurement of the relative escape fraction of galaxies in our sample by C. C. Steidel et al. (2016, in preparation). For the subsample of galaxies with deep LyC spectra-where the sample has been cleaned of foreground contamination based on the ground-based spectroscopy (see Section 2)-these authors measured the ratio of the flux densities at 900 and $1500 \AA$, $\langle S(900) / S(1500)\rangle_{\mathrm{obs}}=0.019 \pm 0.002$. With this measurement, we can invert Equation (16) to compute $\langle S(900) / S(1500)\rangle_{\text {int }}$. To account fully for the measurement and random errors that enter into Equation (16), we performed a Monte Carlo simulation by perturbing each variable in the equation by its associated measurement or random uncertainty. As noted above, the mean combined IGM and CGM attenuation at the mean redshift of the 121 galaxies of $\langle z\rangle=3.05$ is $\left\langle\exp \left[-\tau_{\mathrm{IGM}}(\mathrm{LyC})\right] \times\right.$ $\left.\exp \left[-\tau_{\mathrm{CGM}}(\mathrm{LyC})\right]\right\rangle=0.370 \pm 0.030$. The mean reddening of these galaxies is $\langle E(B-V)\rangle=0.212 \pm 0.003$, assuming the Reddy et al. (2015) dust attenuation curve. For this attenuation curve, $k(1500 \AA)=8.687 \pm 0.200$ as calculated in Paper I.

We performed 10,000 simulations where, each time, we randomly perturbed the IGM+CGM attenuation, the mean reddening, the dust attenuation curve, and the observed relative escape fraction according to normal distributions centered on the mean values with $\sigma$ equal to the associated uncertainties. In addition, we randomly perturbed $f_{\text {cov }}(\mathrm{H} \mathrm{I})$ and $E(B-V)$ for each composite according to their respective uncertainties and fit Equation (15) to these perturbed values. We then calculated $\langle S(900) / S(1500)\rangle_{\text {int }}$ for each of the 10,000 trials. The resulting distributions of $\langle S(900) / S(1500)\rangle_{\text {int }}$ are shown in Figure 12. We find $\langle S(900) / S(1500)\rangle_{\text {int }}=0.199 \pm 0.038$ and $0.240 \pm$ 0.047 assuming the Calzetti et al. (2000) and Reddy et al. (2015) attenuation curves, respectively (with an SMC line of sight extinction in both cases). The simulations indicate that we can use our methodology to constrain the intrinsic 900-to$1500 \AA$ flux density ratio to $\lesssim 20 \%$ random error for the sample of galaxies considered here.

In addition to this random error, there are several sources of systematic error in our estimate of $\langle S(900) / S(1500)\rangle_{\text {int }}$, including the choice of the line-of-sight extinction curve (e.g., SMC or MW) and the choice of the stellar reddening curve (e.g., Reddy et al. 2015 or Calzetti et al. 2000). The magnitude of the bias in $\langle S(900) / S(1500)\rangle_{\text {int }}$ that stems from these effects is $\lesssim 2 \%$ and $\approx 25 \%$, respectively. Additional systematic uncertainty may arise from the choice of IGM prescription. For example, Inoue et al. (2014) find a mean IGM transmission of $\approx 0.5$ for $900 \AA$ photons emitted from a source at $z=3$, a value at the high end of published measurements. On the other hand, the combined IGM+CGM opacity predicted by Rudie et al. (2013) is 0.37. This suggests a systematic uncertainty associated with the IGM prescription of $\approx 30 \%$.

The largest source of systematic uncertainty relates to our inability to fully resolve the $\mathrm{H}$ I absorption. Specifically, our estimate of $f_{\text {cov }}(\mathrm{H} \mathrm{I})$ may be a lower limit as a result of several effects, some of which are discussed in Section 4.3. First, the relatively low spectral resolution of our data may mask the presence of narrow absorption line systems with a higher covering fraction than that deduced from the spectra. Second, if

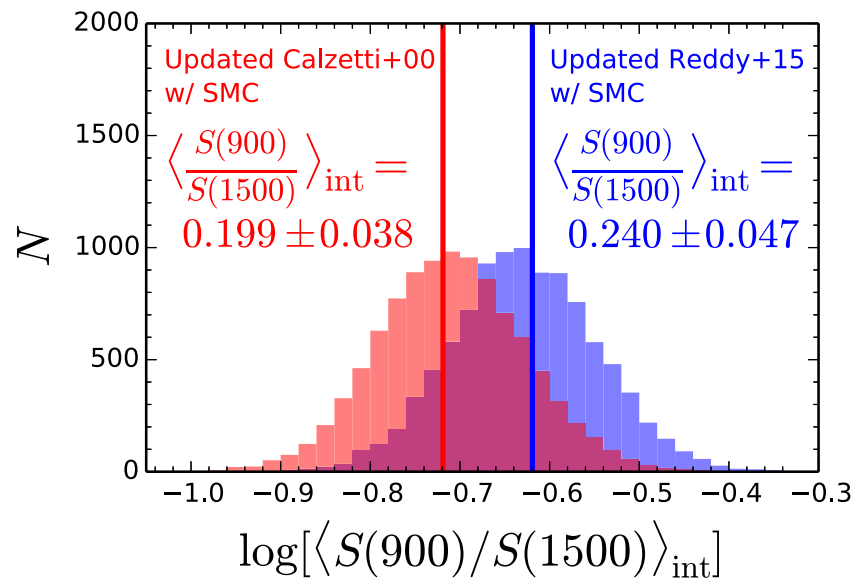

Figure 12. Distribution of the intrinsic average 900-to- $1500 \AA$ flux density ratios calculated using Equation (16) for 10,000 trials. In each trial, we perturbed the attenuation by the IGM+CGM, the mean reddening, the relationship between $f_{\text {cov }}$ and $E(B-V)$, the value of the attenuation curve at $1500 \AA \quad(k(1500))$, and the observed relative escape fraction of $\langle S(900) / S(1500)\rangle_{\mathrm{obs}}=0.019 \pm 0.002$ from C. C. Steidel et al. (2016, in preparation), each according to the random/measurement errors. Shown are the distributions when we assume the updated Reddy et al. (2015) (blue) and Calzetti et al. (2000) (red) attenuation curves for the stellar continuum, and an $\mathrm{SMC}$ extinction curve for the line-of-sight reddening. Indicated are the means (vertical lines) and $1 \sigma$ dispersions of the intrinsic 900-to-1500 $\AA$ flux density ratios based on the 10,000 trials. The distributions obtained with the original versions of the Reddy et al. (2015) and Calzetti et al. (2000) attenuation curves for the reddening of the stellar continuum and/or by assuming a MW extinction curve for the line-of-sight reddening are essentially identical to the distributions shown here.

the different velocity components of the optically thick $\mathrm{H}$ I are not covering the same lines-of-sight, then the integrated covering fraction may be larger than the value found for the bulk of the absorbing gas. Third, we are unable to discern whether non-negligible columns of $\mathrm{H}$ I and dust exist in regions that we would otherwise refer to as "clear" sightlines. Fourth, the ionized gas may not be completely bounded by $\mathrm{H}$ I (i.e., the "average" configuration of the $\mathrm{H}$ I and $\mathrm{H}$ II may be intermediate between the radiation- and density-bounded scenarios). In this latter case, attenuation by dust in the ionized gas may deplete LyC photons before they escape the galaxy. For these reasons, the effective covering fraction, $f_{\text {cov }}^{\text {eff }}(\mathrm{H} \mathrm{I})$, may be larger than the value derived from the composite spectrum and hence the calculated value of $\langle S(900) / S(1500)\rangle_{\text {int }}$ may be a lower limit.

To further evaluate the range of possible $\langle S(900) / S(1500)\rangle_{\text {int }}$ allowed by the data, we compared our estimate with the predictions of several stellar population synthesis models (Figure 13). We considered Bruzual \& Charlot (2003) $0.28 Z_{\odot}$ and $Z_{\odot}$ models with a Salpeter (1955) IMF (power law index of $\alpha=2.35)$ over the mass range $0.1 \leqslant M_{*} \leqslant 100 M_{\odot}$; and the most recent version of the Starburst99 (S99) models which adopt weaker stellar winds relative to the the previous version (Leitherer et al. 2014). We generated S99 models using the Kroupa (2001) IMF with different power law slopes of 2.30, 2.00, and 1.70, over the mass range $0.5 \leqslant M_{*} \leqslant 100 M_{\odot}$, and with a metallicity of $0.14 Z_{\odot}$. Lastly, we consider the "Binary Population and Spectral Synthesis" (BPASS; Stanway et al. 2016) models that include binary evolution, with a power law slope of the IMF of 2.35 over the mass range $0.5 \leqslant M_{*} \leqslant 300 M_{\odot}$, and metallicities of 0.07 and $0.14 Z_{\odot}$. All metallicities assume the solar abundances measured in Asplund et al. (2009). 


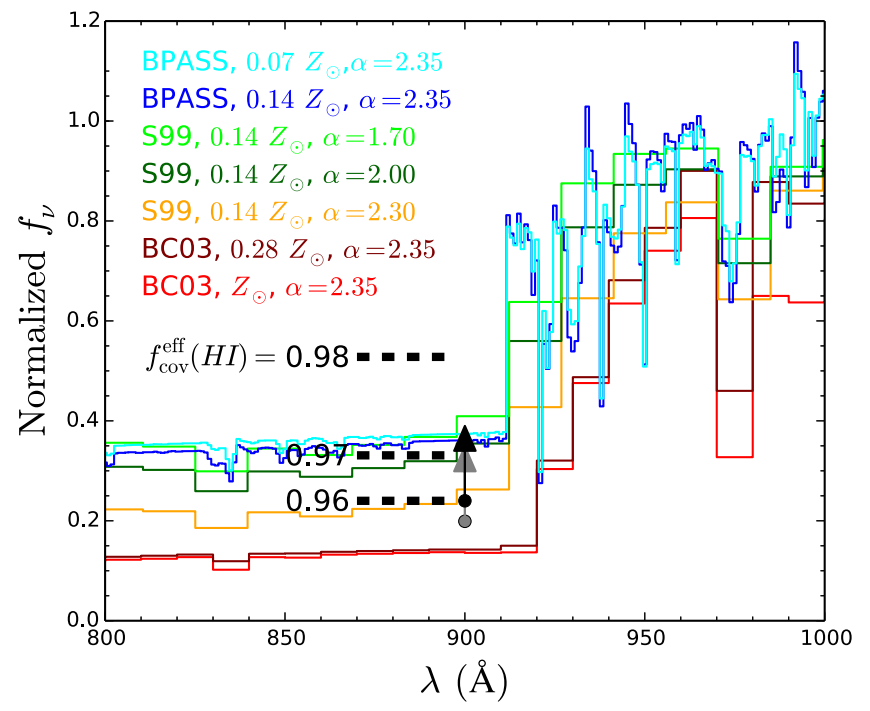

Figure 13. Comparison of different stellar population models, including those of Bruzual \& Charlot (2003), the latest version of Starburst99 (Leitherer et al. 2014), and the latest version of the BPASS models (Stanway et al. 2016) at extreme- to far-UV wavelengths for different metallicities and power law indices $(\alpha)$ of the IMF. All of the models are normalized to have a unity flux density at $1500 \AA$. The limits in the intrinsic 900-to-1500 A flux density ratio, $\langle S(900) / S(1500)\rangle_{\text {int }}$, as inferred from our spectra for an SMC line-of-sight extinction, and the updated Reddy et al. (2015) and Calzetti et al. (2000) stellar attenuation curves are indicated by the black and gray arrows, respectively. The covering fraction deduced from the depth of the $\mathrm{H}$ I lines in the composite spectrum of all galaxies in our sample is $f_{\text {cov }}(\mathrm{H} \mathrm{I})=0.96$. If the "effective" covering fraction is $f_{\text {cov }}^{\text {eff }}=0.97$ and 0.98 , then the limit in $\langle S(900) / S(1500)\rangle_{\text {int }}$ rises to 0.33 and 0.53 , respectively, as indicated by the horizontal dashed lines.

The effect of binary evolution (as in the BPASS models) is to allow efficient mass transfer from a massive star to its (secondary) companion, causing the latter to spin up quickly and, for a low stellar metallicity where the stellar wind is sufficiently weak, retain its angular momentum (Cantiello et al. 2007). Consequently, the secondary becomes fully mixed and undergoes quasi-homogeneous evolution (Yoon \& Langer 2005; Brott et al. 2011; Eldridge \& Stanway 2012) whereby the main sequence lifetime and effective temperature are increased. In such models, the ionizing photon flux per non-ionizing UV photon flux may increase by up to $60 \%$ relative to single star evolution (e.g., Stanway et al. 2016; see also Topping \& Shull 2015). A similar effect can be achieved by decreasing the power law index of the IMF, which results in a larger mass fraction in more massive, hotter stars.

Figure 13 shows the limit on $\langle S(900) / S(1500)\rangle_{\text {int }}$ based on $f_{\text {cov }}(\mathrm{H} \mathrm{I}) \approx 0.96$ derived from the composite spectrum of all 933 galaxies in our sample. Our inference of the gas covering fraction favors an intrinsic LyC-to-UV flux density ratio that is larger than the value predicted from the Bruzual \& Charlot (2003) stellar population models, and one that is consistent with those obtained with the newer versions of the S99 models that adopt weaker massive star stellar winds, a low stellar metallicity, and/or a flatter slope of the IMF, or those models that include the effects of binary evolution. For the reasons stated above, the covering fractions derived here are likely lower limits, and therefore it is instructive to gauge the degree to which the covering fraction may be higher and still be consistent with the predictions of stellar population synthesis models.
In particular, if the effective covering fraction is $f_{\text {cov }}^{\text {eff }}(\mathrm{H} \mathrm{I}) \approx 0.97$ or 0.98 instead of 0.96 , then the inferred $\langle S(900) / S(1500)\rangle_{\text {int }}$ increases from 0.24 to 0.31 and 0.51 , respectively. Note that the latter is $\approx 50 \%$ larger than the value predicted by the low-metallicity BPASS models and/or the low-metallicity and shallow- $\alpha$ IMF S99 models. Thus it is apparent, though ironic, that it is those galaxies with the largest covering fractions (as is the case for the average galaxy at $z \sim 3$ ) which provide the most sensitive constraints on the intrinsic LyC-to-UV flux density ratio, since even small increases in $f_{\text {cov }}(\mathrm{H} \mathrm{I})$ will lead to very large changes in the inferred $\langle S(900) / S(1500)\rangle_{\text {int }}$ (Figure 13). Steidel et al. (2016) suggest that stellar population models that include the effects of binary evolution are required to simultaneously reproduce the rest-frame far-UV continuum, stellar, and nebular features, and the rest-frame optical nebular emission line strengths measured for galaxies at $z \sim 2$. If we adopt such models as the most appropriate, then it implies that our estimate of $f_{\text {cov }}(\mathrm{H} \mathrm{I})$ for typical galaxies at $z \sim 3$ is not biased low by more than $\approx 1 \%$. Stated another way, the combined effects of additional $\mathrm{H} \mathrm{I}$ absorption and dust attenuation cannot deplete more than $25 \%$ of the photons exiting through what we would otherwise refer to as "clear" sightlines based on the depths of the $\mathrm{H}$ I lines in the composite spectra.

This analysis underscores the fact that if the simple geometry of Figure 3 is valid on average for an ensemble of galaxies, then deep UV spectroscopy for large samples of typical starforming galaxies at high redshift should enable tight constraints on the intrinsic LyC-to-UV flux density ratio. Hence, our framework provides an alternative and powerful independent method of constraining the intrinsic LyC-to-UV flux density ratio, which is currently one of the most uncertain parameters entering into the calculation of the absolute escape fraction of ionizing photons in high-redshift galaxies.

\subsubsection{Deviations from the Assumed Geometry}

The geometry depicted in Figure 3 may not apply for all galaxies, and, in reality, more complicated configurations of the dust, gas, and stars may be expected. In this context, the model described here provides a useful benchmark to investigate changes in geometry and hence alternate pathways for the escape of ionizing radiation. To illustrate this, we show in Figure $14\langle S(900) / S(1500)\rangle_{\text {obs }}$ as a function of $E(B-V)$ assuming the fiducial model for the SMC extinction curve. We have assumed $\langle S(900) / S(1500)\rangle_{\text {int }}=0.35$, a value typical of the BPASS models and the S99 models with a flat IMF slope, and IGM and CGM opacities relevant for galaxies at $z=3.05$. Direct measurements of $\langle S(900) / S(1500)\rangle_{\mathrm{obs}}$ and $\langle E(B-V)\rangle$ for an ensemble of galaxies may be used to ascertain the mechanisms by which LyC photons escape galaxies. For example, if these average values lie in the green region, then it suggests that, at least on average, those galaxies have either a smaller intrinsic 900-to- $1500 \AA$ flux density ratio than the one we have adopted here, or that the "uncovered" portions of the continuum are not completely free of dust and/or gas. Similarly, objects whose average values place them in the purple region are objects that may have a larger intrinsic 900to- $1500 \AA$ flux density ratio or optically thin neutral gas that allows the escape of additional LyC photons. Finally, galaxies whose average values lie in the cyan region must have a larger intrinsic 900-to-1500 $\AA$ flux density ratio on average than the one assumed here. Further discrimination of the mechanisms 


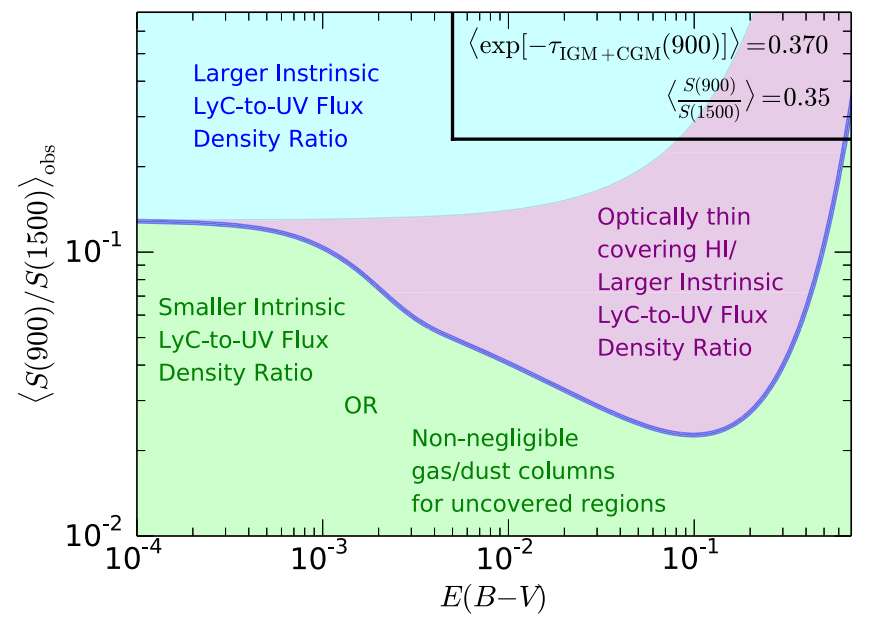

Figure 14. Same as Figure 11, where only the fiducial model for the SMC extinction curve (blue line) is shown for simplicity, and we have assumed $\langle S(900) / S(1500)\rangle_{\text {int }}=0.35$. As before, an IGM+CGM opacity appropriate for galaxies at $z=3.05$ is assumed. A measured $\langle S(\mathrm{LyC}) / S(\mathrm{UV})\rangle_{\mathrm{obs}}$ and $\langle E(B-V)\rangle$ in the green shaded for an ensemble of galaxies implies either $\langle S(900) / S(1500)\rangle_{\text {int }}<0.35$, or that the "uncovered" portions of the galaxies' spectra have a non-negligible column density of gas and/or dust. Galaxies with average values that place them in the purple region may have a larger intrinsic 900-to-1500 $\AA$ flux density ratio or outflowing neutral gas that is optically thin (allowing for the leakage of additional LyC photons). The boundary between the cyan and purple regions is defined by setting $f(900)_{\mathrm{abs}}=1$, so that if the average values place the galaxies in the cyan region, then such galaxies must have a larger intrinsic 900 -to- $1500 \AA$ flux density ratio than the one assumed here.

by which LyC photons escape galaxies, or of the value of the intrinsic LyC-to-UV flux density ratio, can be accomplished with deep spectroscopy, such as that presented here. From this spectroscopy, we have found that typical $z \sim 3$ galaxies can be characterized by optically thick outflowing neutral $\mathrm{HI}$, a finding that already narrows the range of possible avenues by which ionizing radiation can escape these galaxies.

\section{CONCLUSIONS}

In this paper, we have modeled the rest-frame far-UV composite spectra of $933 \mathcal{R}<25.5$ LBGs at $z \sim 3,121$ of which have very deep spectroscopic covering at $\lambda=850-1300 \AA$, in order to investigate the relationship between UV reddening, neutral gas covering fraction, and the ionizing photon escape fraction. This analysis is facilitated by our large sample of galaxies which allows us to average over stochastic variations in the $\operatorname{Ly} \alpha$ forest along different sightlines to $z \sim 3$ galaxies, and thus securely analyze the $\mathrm{H} \mathrm{I}$ absorption lines in these galaxies. Our main conclusions are as follows.

1. The composite spectra of $z \sim 3$ galaxies show evidence for a partial covering of optically thick outflowing neutral hydrogen. Specifically, the gas is (a) blueshifted by $\approx 300 \mathrm{~km} \mathrm{~s}^{-1}$; (b) exhibits damping wings in the Lyman series lines, including Ly $\alpha$, signifying high-column density, $\log \left[N(\mathrm{H} \mathrm{I}) / \mathrm{cm}^{-2}\right] \gtrsim 20.3$; and (c) Lyman series lines including $\mathrm{Ly} \beta, \mathrm{Ly} \gamma$, and $\mathrm{Ly} \delta$ that do not reach zero flux density at the line cores, implying the presence of unabsorbed stellar continuum. This result is robust against spectral resolution and redshift uncertainties, and is based upon a careful isolation of foreground contaminants.
2. To investigate trends between reddening and gas covering fraction, we divided our sample into bins of $E(B-V)$ (measured from the stellar continuum) and constructed composites for galaxies in each of these bins. We modeled the composite spectra, allowing for a nonunity covering fraction of $\mathrm{H}$ I and dust-a configuration that arises from an increase in ISM porosity due to stellar feedback processes; and including both stellar and nebular continuum emission, as well as unresolved absorption from the $\mathrm{H}_{2}$ Lyman-Werner bands. The derived H I covering fractions vary from 92 to $97 \%$, over a range of continuum reddening from $E(B-V)=0.098$ to 0.292 . The modeling results imply that galaxies with redder UV continua have a larger covering fraction of $\mathrm{H} \mathrm{I}$ and dust, with a higher line-ofsight dust column density.

3. The absolute escape fraction of $\operatorname{Ly} \alpha$ emission, as measured through a spectroscopic slit, correlates inversely with neutral gas covering fraction. This suggests that the Ly $\alpha$ photons escape preferentially through sightlines that have been cleared of gas and dust. Separately, the neutral gas covering fractions deduced from saturated low-ionization interstellar absorption lines, such as Si II $\lambda \lambda 1260,1527 \AA$, correlate with, but systematically underpredict by a factor of $\simeq 1.5$, those derived from the $\mathrm{H}$ I lines. Thus, inferences of gas covering fractions based on low-ionization interstellar absorption lines must be viewed with caution. Moreover, this result suggests that a significant fraction of outflowing $\mathrm{H}$ I may be metal-poor. The fact that the "covering fractions" inferred from the low-ionization interstellar absorption lines appear to rise rapidly with a small increase in $f_{\text {cov }}(\mathrm{H} \mathrm{I})$, together with our finding that the line-of-sight extinction increases in tandem with the reddening of the stellar continuum, suggests that the outflowing material becomes progressively enriched with metals for galaxies with larger $f_{\text {cov }}(\mathrm{H} \mathrm{I})$ and redder UV continua.

4. We discuss our results in the context of the escape of ionizing radiation at high redshift. The partial covering fraction of optically thick $\mathrm{H}$ I implies that the depletion of ionizing photons is dominated by photoelectric absorption rather than dust attenuation, and that ionizing photons escape typical galaxies at $z \sim 3$ through clear sightlines in the ISM.

5. Using a physically motivated functional form for the relationship between continuum reddening and neutral gas covering fraction, we establish a relationship between the absolute and relative escape fraction of ionizing radiation and continuum reddening. Our model may be used to predict the average escape fractions of ionizing photons for an ensemble of galaxies with some average $E(B-V)$ and an assumed intrinsic LyC-to-UV flux density ratio. Alternatively, direct measurements of the ionizing escape fraction may be used to place constraints on the intrinsic LyC-to-UV flux density ratio. Based on a recent measurement of $\langle S(900) / S(1500)\rangle_{\text {obs }}$ for the subsample of galaxies with deep far-UV spectra analyzed here, we infer $\langle S(900) / S(1500)\rangle_{\text {int }} \gtrsim 0.20$. This lower limit is larger than the prediction from the Bruzual \& Charlot (2003) models, and generally favors stellar population models that include weaker stellar winds, a flatter slope of the IMF, and/or binary evolution. 
We have demonstrated how our model may be used to provide powerful constraints on the intrinsic ionizing spectrum of high-redshift galaxies, and, additionally, how galaxies with large covering fractions and associated large reddening may be used as sensitive probes of the intrinsic LyC-to-UV flux density ratio. Aside from providing constraints on the production efficiency of ionizing photons, we have discussed how our model may also be used to assess the pathways by which such photons escape galaxies.

Our analysis points to several future avenues of investigation. Coupling deep rest-frame UV spectroscopy of highredshift galaxies (such as that presented here) with multi-band $H S T$ imaging will allow us to construct the cleanest possible sample of galaxies - uncontaminated by foreground interlopers based on high spatial resolution multi-component photometric redshift analysis (Mostardi et al. 2015) and the identification of spectroscopic blends-with which to measure the ionizing escape fraction. The high spatial resolution provided by $H S T$ has other potential benefits in terms of clarifying the escape of ionizing radiation. Specifically, a consequence of a partial covering fraction of $\mathrm{H} \mathrm{I}$ is an inhomogeneous spatial distribution of LyC emission relative to the non-ionizing UV continuum. While resolving individual sightlines in these high-redshift galaxies is far beyond HST's capabilities, a "clumpy" distribution of LyC emission that is resolved with $H S T$ to be disjoint from the morphology of the non-ionizing UV continuum may still indicate the signature of a porous ISM where LyC photons only escape through clear lines-of-sight.

More precise measurements of, or limits on, the neutral gas covering fractions may be achieved with higher resolution spectroscopy for large samples of galaxies, possible with the next-generation of instruments and $\gtrsim 30 \mathrm{~m}$ telescopes. In the more immediate future, rest-frame optical nebular emission line spectroscopy will enable systemic redshift measurements that can be used to analyze the $\mathrm{H}$ I line profiles from composite spectra in a way that is less affected by redshift uncertainties. The combination of rest-frame optical nebular spectroscopy, rest-frame UV continuum spectroscopy, and our model for the escape of ionizing radiation together will make it possible to construct a consistent picture of the massive and ionizing stellar populations in high-redshift galaxies. Lastly, the models presented here will benefit greatly from future deep spectroscopy of bluer galaxies and/or those detected with strong Ly $\alpha$ emission to extend the dynamic range of continuum reddening probed in our study. With such spectroscopy, we will be in a position to evaluate the column densities, line-of-sight reddening, and covering fractions for the types of galaxies that are likely to contribute significantly to the budget of ionizing photons at high redshift. The framework presented in this paper will be central to the interpretation of the ionizing escape fractions of galaxies at the reionization epoch, where the prohibitively thick IGM precludes the detailed far-UV measurements presented here.

N.A.R. is supported by an Alfred P. Sloan Research Fellowship, and acknowledges the visitors program at the Institute of Astronomy in Cambridge, UK, where part of this research was conducted. C.C.S. acknowledges NSF grants AST-0908805 and AST-1313472. MB acknowledges support of the Serbian MESTD through grant ON176021. NAR acknowledges Gwen Rudie for useful discussions. We are grateful to the anonymous referee whose comments led to significant improvements in the clarity, content, and presentation of the analysis and results. We wish to extend special thanks to those of Hawaiian ancestry on whose sacred mountain we are privileged to be guests. Without their generous hospitality, the observations presented herein would not have been possible.

\section{REFERENCES}

Alexandroff, R. M., Heckman, T. M., Borthakur, S., Overzier, R., \& Leitherer, C. 2015, ApJ, 810, 104

Armus, L., Heckman, T. M., Weaver, K. A., \& Lehnert, M. D. 1995, ApJ, 445,666

Asplund, M., Grevesse, N., Sauval, A. J., \& Scott, P. 2009, ARA\&A, 47, 481 Bagetakos, I., Brinks, E., Walter, F., et al. 2011, AJ, 141, 23

Bolton, J. S., \& Haehnelt, M. G. 2007, MNRAS, 382, 325

Bolton, J. S., Haehnelt, M. G., Viel, M., \& Springel, V. 2005, MNRAS, 357, 1178

Borthakur, S., Momjian, E., Heckman, T. M., et al. 2014, ApJ, 795, 98

Bouwens, R. J., Illingworth, G. D., Oesch, P. A., et al. 2015, ApJ, 803, 34

Bouwens, R. J., Thompson, R. I., Illingworth, G. D., et al. 2004, ApJL, 616, L79

Bridge, C. R., Teplitz, H. I., Siana, B., et al. 2010, ApJ, 720, 465

Brott, I., de Mink, S. E., Cantiello, M., et al. 2011, A\&A, 530, A115

Bruzual, G., \& Charlot, S. 2003, MNRAS, 344, 1000

Bunker, A. J., Wilkins, S., Ellis, R. S., et al. 2010, MNRAS, 409, 855

Calzetti, D., Armus, L., Bohlin, R. C., et al. 2000, ApJ, 533, 682

Cantiello, M., Yoon, S.-C., Langer, N., \& Livio, M. 2007, A\&A, 465, L29

Clarke, C., \& Oey, M. S. 2002, MNRAS, 337, 1299

Cowie, L. L., Barger, A. J., \& Trouille, L. 2009, ApJ, 692, 1476

de Barros, S., Vanzella, E., Amorín, R., et al. 2016, A\&A, 585, 51

Deharveng, J.-M., Buat, V., Le Brun, V., et al. 2001, A\&A, 375, 805

Dijkstra, M., Gronke, M., \& Venkatesan, A. 2016, arXiv:1604.08208

Duncan, K., \& Conselice, C. J. 2015, MNRAS, 451, 2030

Eldridge, J. J., \& Stanway, E. R. 2009, MNRAS, 400, 1019

Eldridge, J. J., \& Stanway, E. R. 2012, MNRAS, 419, 479

Ellis, R. S., McLure, R. J., Dunlop, J. S., et al. 2013, ApJL, 763, L7

Fan, X., Narayanan, V. K., Lupton, R. H., et al. 2001, AJ, 122, 2833

Finkelstein, S. L., Papovich, C., Ryan, R. E., et al. 2012, ApJ, 758, 93

Fontanot, F., Cristiani, S., \& Vanzella, E. 2012, MNRAS, 425, 1413

Giallongo, E., Grazian, A., Fiore, F., et al. 2015, A\&A, 578, A83

Glikman, E., Djorgovski, S. G., Stern, D., et al. 2011, ApJL, 728, L26

Gnedin, N. Y., Kravtsov, A. V., \& Chen, H.-W. 2008, ApJ, 672, 765

Grazian, A., Castellano, M., Fontana, A., et al. 2012, A\&A, 547, A51

Grimes, J. P., Heckman, T., Aloisi, A., et al. 2009, ApJS, 181, 272

Hayes, M., Schaerer, D., Östlin, G., et al. 2011, ApJ, 730, 8

Heckman, T. M., Borthakur, S., Overzier, R., et al. 2011, ApJ, 730, 5

Hurwitz, M., Jelinsky, P., \& Dixon, W. V. D. 1997, ApJL, 481, L31

Inoue, A. K., Iwata, I., \& Deharveng, J.-M. 2006, MNRAS, 371, L1

Inoue, A. K., Shimizu, I., Iwata, I., \& Tanaka, M. 2014, MNRAS, 442, 1805

Iwata, I., Inoue, A. K., Matsuda, Y., et al. 2009, ApJ, 692, 1287

Izotov, Y. I., Orlitová, I., Schaerer, D., et al. 2016a, Natur, 529, 178

Izotov, Y. I., Schaerer, D., Thuan, T. X., et al. 2016b, MNRAS, 461, 3683

Jones, T. A., Ellis, R. S., Schenker, M. A., \& Stark, D. P. 2013, ApJ, 779, 52 Kennicutt, R. C. 1998, ARA\&A, 36, 189

Kornei, K. A., Shapley, A. E., Erb, D. K., et al. 2010, ApJ, 711, 693

Kroupa, P. 2001, MNRAS, 322, 231

Kunth, D., Mas-Hesse, J. M., Terlevich, E., et al. 1998, A\&A, 334, 11

Lehnert, M. D., \& Heckman, T. M. 1995, ApJS, 97, 89

Leitet, E., Bergvall, N., Hayes, M., Linné, S., \& Zackrisson, E. 2013, A\&A, 553, A106

Leitherer, C., Ekström, S., Meynet, G., et al. 2014, ApJS, 212, 14

Leitherer, C., Ferguson, H. C., Heckman, T. M., \& Lowenthal, J. D. 1995, ApJL, 454, L19

Leitherer, C., Hernandez, S., Lee, J. C., \& Oey, M. S. 2016, ApJ, 823, 64

Leitherer, C., Ortiz Otálvaro, P. A., Bresolin, F., et al. 2010, ApJS, 189, 309

Leitherer, C., Schaerer, D., Goldader, J. D., et al. 1999, ApJS, 123, 3

Levesque, E. M., Leitherer, C., Ekstrom, S., Meynet, G., \& Schaerer, D. 2012 , ApJ, 751, 67

Lynds, C. R., \& Sandage, A. R. 1963, ApJ, 137, 1005

Ma, X., Hopkins, P. F., Kasen, D., et al. 2016, MNRAS, 459, 3614

Madau, P., \& Haardt, F. 2015, ApJL, 813, L8

Madau, P., Haardt, F., \& Rees, M. J. 1999, ApJ, 514, 648

McDonald, P., \& Miralda-Escudé, J. 2001, ApJL, 549, L11

McLure, R. J., Dunlop, J. S., Cirasuolo, M., et al. 2010, MNRAS, 403, 960 
Mostardi, R. E., Shapley, A. E., Nestor, D. B., et al. 2013, ApJ, 779, 65

Mostardi, R. E., Shapley, A. E., Steidel, C. C., et al. 2015, ApJ, 810, 107

Nestor, D. B., Shapley, A. E., Kornei, K. A., Steidel, C. C., \& Siana, B. 2013, ApJ, 765, 47

Nestor, D. B., Shapley, A. E., Steidel, C. C., \& Siana, B. 2011, ApJ, 736, 18

Oesch, P. A., Bouwens, R. J., Illingworth, G. D., et al. 2010, ApJL, 709, L16

Oesch, P. A., Bouwens, R. J., Illingworth, G. D., et al. 2013, ApJ, 773, 75

Oey, M. S., Groves, B., Staveley-Smith, L., \& Smith, R. C. 2002, AJ, 123, 255

Oke, J. B., Cohen, J. G., Carr, M., et al. 1995, PASP, 107, 375

O'Meara, J. M., Prochaska, J. X., Worseck, G., Chen, H.-W., \& Madau, P. 2013, ApJ, 765, 137

Putman, M. E., Peek, J. E. G., \& Joung, M. R. 2012, ARA\&A, 50, 491

Razoumov, A. O., \& Sommer-Larsen, J. 2010, ApJ, 710, 1239

Reddy, N., Dickinson, M., Elbaz, D., et al. 2012, ApJ, 744, 154

Reddy, N. A., Kriek, M., Shapley, A. E., et al. 2015, ApJ, 806, 259

Reddy, N. A., \& Steidel, C. C. 2009, ApJ, 692, 778

Reddy, N. A., Steidel, C. C., Pettini, M., et al. 2008, ApJS, 175, 48

Reddy, N. A., Steidel, C. C., Pettini, M., et al. 2016, ApJ, 828, 108

Rivera-Thorsen, T. E., Hayes, M., Östlin, G., et al. 2015, ApJ, 805, 14

Rix, S. A., Pettini, M., Leitherer, C., et al. 2004, ApJ, 615, 98

Robertson, B. E., Ellis, R. S., Dunlop, J. S., McLure, R. J., \& Stark, D. P. 2010, Natur, 468, 49

Robertson, B. E., Ellis, R. S., Furlanetto, S. R., \& Dunlop, J. S. 2015, ApJL, 802, L19

Rudie, G. C., Steidel, C. C., Shapley, A. E., \& Pettini, M. 2013, ApJ, 769, 146

Salpeter, E. E. 1955, ApJ, 121, 161

Shapiro, P. R., \& Field, G. B. 1976, ApJ, 205, 762

Shapley, A. E., Steidel, C. C., Pettini, M., \& Adelberger, K. L. 2003, ApJ, 588,65

Shapley, A. E., Steidel, C. C., Pettini, M., Adelberger, K. L., \& Erb, D. K. 2006, ApJ, 651, 688
Shapley, A. E., Steidel, C. C., Strom, A. L., et al. 2016, ApJ, 826, 24

Siana, B., Shapley, A. E., Kulas, K. R., et al. 2015, ApJ, 804, 17

Siana, B., Teplitz, H. I., Chary, R.-R., Colbert, J., \& Frayer, D. T. 2008, ApJ, 689,59

Siana, B., Teplitz, H. I., Colbert, J., et al. 2007, ApJ, 668, 62

Siana, B., Teplitz, H. I., Ferguson, H. C., et al. 2010, ApJ, 723, 241

Silk, J. 1997, ApJ, 481, 703

Stanway, E. R., Eldridge, J. J., \& Becker, G. D. 2016, MNRAS, 456, 485

Stark, D. P., Ellis, R. S., Richard, J., et al. 2007, ApJ, 663, 10

Steidel, C. C., Adelberger, K. L., Giavalisco, M., Dickinson, M., \& Pettini, M. 1999, ApJ, 519, 1

Steidel, C. C., Adelberger, K. L., Shapley, A. E., et al. 2003, ApJ, 592, 728

Steidel, C. C., Bogosavljević, M., Shapley, A. E., et al. 2011, ApJ, 736, 160

Steidel, C. C., Erb, D. K., Shapley, A. E., et al. 2010, ApJ, 717, 289

Steidel, C. C., Pettini, M., \& Adelberger, K. L. 2001, ApJ, 546, 665

Steidel, C. C., Shapley, A. E., Pettini, M., et al. 2004, ApJ, 604, 534

Steidel, C. C., Strom, A. L., Pettini, M., et al. 2016, ApJ, 826, 159

Strickland, D. K., Heckman, T. M., Colbert, E. J. M., Hoopes, C. G., \& Weaver, K. A. 2004, ApJ, 606, 829

Topping, M. W., \& Shull, J. M. 2015, ApJ, 800, 97

Trainor, R. F., Steidel, C. C., Strom, A. L., \& Rudie, G. C. 2015, ApJ, 809,89

Vanzella, E., Giavalisco, M., Inoue, A. K., et al. 2010a, ApJ, 725, 1011

Vanzella, E., Guo, Y., Giavalisco, M., et al. 2012, ApJ, 751, 70

Vanzella, E., Siana, B., Cristiani, S., \& Nonino, M. 2010b, MNRAS, 404, 1672

Welty, D. E., Xue, R., \& Wong, T. 2012, ApJ, 745, 173

Wofford, A., Leitherer, C., \& Salzer, J. 2013, ApJ, 765, 118

Yan, H., \& Windhorst, R. A. 2004, ApJL, 600, L1

Yoon, S.-C., \& Langer, N. 2005, A\&A, 443, 643

Zackrisson, E., Inoue, A. K., \& Jensen, H. 2013, ApJ, 777, 39 